Revue européenne des sciences sociales

European Journal of Social Sciences

XXXIX-120 | 2001

Autour de l'épistémologie de la sociologie et de la sociologie des sciences: des débats, un réexamen

\title{
La forme des preuves dans les sciences historiques
}

Jean-Claude Passeron

\section{OpenEdition}

1 Journals

Édition électronique

URL : http://journals.openedition.org/ress/655

DOI : $10.4000 /$ ress. 655

ISSN : 1663-4446

Éditeur

Librairie Droz

Édition imprimée

Date de publication : 1 juillet 2001

Pagination : $31-76$

ISBN : 2-600-00610-9

ISSN : 0048-8046

Référence électronique

Jean-Claude Passeron, "La forme des preuves dans les sciences historiques », Revue européenne des sciences sociales [En ligne], XXXIX-120 | 2001, mis en ligne le 11 décembre 2009, consulté le 30 avril 2019. URL : http://journals.openedition.org/ress/655; DOI : 10.4000/ress.655 


\section{LA FORME DES PREUVES DANS LES SCIENCES HISTORIQUES*}

Qu'est-ce que «parler veut dire» en sociologie? Autrement dit, s'agissant du discours d'une science: de quels critères de vérité ou de fausseté ses énoncés sontils susceptibles? Restreignons cette question épistémologique à l'examen des méthodes utilisées pour mettre en rapport des énoncés de causalité avec des énoncés d'observation: que signifie «faire preuve» dans une science historique? C'est la définition minimum d'une science que de dire qu'on y prouve quelque chose plutôt que rien.

Comment la sociologie fait-elle la preuve des analyses qu'elle a accumulées pendant les deux siècles qui l'ont vue se constituer comme science empirique en se dégageant des sciences normatives, puis se développer et se ramifier en multipliant ses échanges avec les autres sciences sociales? Je ne me demande donc pas ici comment elle devrait procéder pour calquer la logique de ses explications sur celle des sciences exactes, en mesurant la valeur de ses preuves à l'étalon des méthodes spécialisées qu'emploient ces dernières; je n'essaie pas non plus d'évaluer sa portée anthropologique ou son utilité politique à l'aune conceptuelle des systèmes philosophiques. Je me demande encore moins si et comment la sociologie pourrait dans l'avenir transformer son identité scientifique pour se conformer à un de ces programmes de re-fondation théorique qu'on voit se succéder bon an mal an: chaque décade a vu paraître dans les sciences sociales au moins plusieurs Manifestes ou Discours de la méthode, un temps moteurs de l'invention théorique, mais vite émoussés dans leur ambition d'avoir réussi une révolution scientifique.

Je me demande simplement quelle est la forme des preuves par lesquelles opère la sociologie dans les textes et discours issus de ses travaux effectifs de recherche. Comment les sociologues prouvent-t-ils les conclusions dont ils affirment la «vérité » au terme de leurs raisonnements? Voilà une question de pure description - une question d'anatomie et de physiologie de la preuve, si l'on préfère - dès lors qu'on accepte comme un fait d'histoire des sciences que la sociologie existe. Et qu'elle existe comme science, puisqu'elle «explique» et fait « comprendre» quelque chose de l'histoire des groupes humains par des méthodes qui, si diverses soient-elles, impliquent toujours un raisonnement comparatif: les règles grammaticales et sémantiques d'inférence qui permettent d'appliquer un

* Ce texte a fait l'objet d'une conférence à l'Université de tous les savoirs (2 avril 2000, Paris). Un bref résumé en a été publié dans le troisième volume de la série des conférences UTLS sous le titre: «Le raisonnement sociologique: la preuve et le contexte», in Eric Michaud (sous dir. de), Qu'est-ce que la société? Paris, Odile Jacob Ed., pp. 38-51. 
raisonnement comparatif à l'explication du monde historique sont, en effet, les mêmes en tout esprit scientifique, par quelque méthode et en quelque discipline que celui-ci se spécifie.

La sociologie n'est pas ce que la plupart des sociologues en disent. Ni lorsque, bâtisseurs de systèmes, ils la vantent orgueilleusement comme un savoir totalisant de la société, une découverte des «lois d'airain» (matérielles ou symboliques) qui expliqueraient «en dernière analyse» son devenir et son avenir. Ni non plus lorsque, étourdiment «physicalistes », ils la pressent de devenir enfin, à grand renfort de mathématiques, de formalisation ou par l'emprunt à l'économie de ses modèles de « rationalité d'action », une «science dure » comme d'autres, quel que soit le prix à payer, pour cette «purification» théorique, en termes de compréhension historique. Ni, encore moins, lorsque, acceptant de passer sous les fourches caudines de la critique «scientiste», ils se résignent à considérer leur discipline comme une «science molle», abdication scientifique où le masochisme épistémologique a moins de part que la fausse modestie: ce sont les mêmes qu'on voit se consoler bien vite de cette position subalterne en revendiquant, l'œil humide de nostalgie, la vocation «humaniste» des sciences de l'homme. L'auto-dépréciation scientifique fait alors le lit de l'auto-glorification philosophique.

\section{RETOUR À UNE ÉPISTÉMOLOGIE DESCRIPTIVE}

Pour juger de la scientificité d'une pratique, décrivons-la. Autrement dit, cessons d'entasser et d'agiter des questions préalables: "Qu'est-ce que le fait social?»- La sociologie a-t-elle une «utilité sociale»? Favorise-t-elle la contestation ou le conservatisme? La diversité contradictoire de ses analyses n'encouragerait-elle pas plutôt le désengagement politique? Ou encore: comment définir la «bonne méthode»? Quelle est la «bonne théorie» dans laquelle il faudrait s'installer et peiner, comme dans un purgatoire préliminaire, pour faire ensuite une sociologie vraiment scientifique?

Contentons-nous, pour identifier le métier que nous faisons, de décrire la forme de raisonnement qu'il implique, c'est-à-dire la forme particulière d'argumentation scientifique que les sociologues ont tous en commun de pratiquer, si on accepte d'oublier un moment leurs conflits théoriques et leurs polémiques métasociologiques. Demandons-nous seulement: comment les sociologues justifientils leurs inférences? Essayons d'identifier les règles de leurs actes de probation par la seule description des opérations qu'ils utilisent vraiment dans leurs raisonnements. En fait, on se trouve avoir décrit du même coup l'argumentation de tous les chercheurs qui travaillent dans une science historique, puisque - comme on va le constater - la parenté épistémologique entre toutes les sciences historiques est au principe de leurs contraintes méthodologiques communes. Sur quelles opérations logiques s'appuient donc les chercheurs de ces disciplines lorsqu'ils affirment ou nient la vérité d'une description ou l'exactitude d'un récit, lorsqu'ils affirment ou nient la force probante d'une comparaison ou la portée d'un calcul statistique, lorsqu'ils affirment ou refusent la nécessité d'une conséquence?

Décrire, c'est toujours décrire par différence, en recherchant les différences pertinentes par rapport à la question d'identification que l'on pose. La phonologie 
moderne s'est fondée sur la décision de recourir à la «commutation » des signifiés pour construire le «tableau phonologique» des traits phonétiques pertinents, et de ceux-là seulement, qui séparent les uns des autres les «phonèmes » dont la distinction par les locuteurs permet l'intercompréhension du sens des mots dans l'utilisation d'une «langue naturelle» particulière. De la même manière, l'épistémologie d'une science ne peut avoir d'autre tâche que de décrire les éléments constitutifs du discours explicatif qui, dans cette science, sont pertinents pour l'identification de ses procédures de preuve par différence avec d'autres. Depuis plus d'un siècle, il n'a pas manqué de sociologues ambitieux pour tenter - toujours en vain, on le voit après coup - de reconstruire, chacun pour son propre compte, le système des définitions et des preuves de la sociologie sous la forme d'un paradigme unifié, comme il s'en trouve dans l'histoire des sciences physiques: exemple fascinant de l'unité théorique d'une cité scientifique. D'où, à défaut de pouvoir l'imiter, la tentation de la singer. L'état de «science normale» est défini par Kuhn ${ }^{1}$ comme celui qui, entre deux révolutions scientifiques, procure à l'ensemble des chercheurs un «paradigme» fort, c'est-à-dire un cadre théorique, unique ou prédominant, dans lequel opèrent en toute cohérence les raisonnements reconnus comme probants par tous les chercheurs. Pourquoi est-il si difficile d'admettre que l'intelligibilité des phénomènes historiques que procure la sociologie ne relève pas d'une telle «normalité» théorique? Cette spécificité méthodologique n'est ni une grâce ni une disgrâce.

L'histoire des sciences nous fait en effet constater que toute une série de propriétés de la preuve - comme l'universalité des assertions, la nécessité logique de la déduction dans une «démonstration», ou la «réfutabilité » (falsifiability) d'une proposition universelle dans les «théories empiriques» construites par les sciences expérimentales - n'ont jamais pu être transposées telles quelles dans une science sociale. Décrire le «raisonnement sociologique», c'est d'abord identifier les propriétés logiques par lesquelles sa manière de prouver se distingue de la nécessité d'une « démonstration » mathématique ou de la formulation d'une «loi » universelle. Les théories d'une science expérimentale permettent de déduire d'une hypothèse la forme, nécessairement probante, de ses tests empiriques : c'est-à-dire d'en déduire une classe d' "énoncés d'observation» susceptibles d'être, dans tous les cas où ils se verraient vérifiés, les «falsificateurs» virtuels de l'hypothèse. Cette tâche de démarcation logique est une tâche épistémologique. Décrire sociologiquement une science ne suffit jamais, en effet, à identifier l'univers logique de ses raisonnements: c'est seulement, comme pour tout autre pratique sociale, expliquer son fonctionnement et ses fonctions en les rapportant à leurs conditions historiques d'exercice. Mais l'épistémologie d'une science ne nous livre pas le même bilan explicatif que sa sociologie. Décrire épistémologiquement une science c'est expliciter les principes qui fondent la consistance sémantique et les limites «référentielles» de ce qu'elle affirme et prouve; disons plus brièvement: expliciter les principes de son espace assertorique.

T. Kuhn, La structure des révolutions scientifiques (1 $1^{\text {rè }}$ éd.,1970), trad. frçse, Paris, Flammarion, 1976. 


\section{L'espace logique et l'espace assertorique d'un discours}

Le langage dans lequel une science formule ses assertions n'est que la forme, toujours particularisée par ses constructions d'objet, de «l'espace logique» propre à tout langage qui entreprend de parler d'un monde quelconque: c'est cet «espace» que décrivait Wittgenstein dans son Tractatus logico-philosophicus ${ }^{2}$. Dans la description d'une science il faut toujours en revenir à la description de l' espace logique où se fonde sémantiquement la cohérence de ses "vérités »: tout langage partagé de description du monde suppose que des locuteurs comprennent de la même manière ce que signifie l'affirmation, inhérente à chacune de ses assertions, qu'un énoncé peut y être pensé comme vrai ou faux.

Dans le Tractatus Wittgenstein avait pour but de formuler l'interdépendance entre les règles et les définitions d'un discours assertant sur un monde logique qui ne contiendrait que des «propositions» et des «faits». Les «propositions» de la description logique proposée par le Tractatus ne se «démontrent» pas comme des théorèmes par une déduction qui irait de l'une à l'autre. Comme le dit Wittgenstein, elles ne peuvent que «montrer», en l'explicitant, le sens de la correspondance entre les «faits» et les «propositions», qui n'est évidemment pas celui de «l'adéquation » entre «l'idée» et la «chose» que supposait la métaphysique classique. Ainsi que le commentait Russell dans la Préface qu'il donnait à ce Traité, on peut toujours «représenter» des faits ou des propositions par des symboles susceptibles d'entrer dans un calcul logique (dont le déroulement est par définition indifférent au sens empirique qu'ils sont susceptibles de revêtir dans le monde empirique); mais on ne peut représenter dans un langage formalisé le rapport entre des faits et des propositions, c'est-à-dire le rapport entre le monde et le langage qui en parle. «Le monde est composé de faits, non de choses », comme oblige à le préciser la Proposition 1.1 du Tractatus, dès lors qu'on a posé dans la Proposition $\mathbf{1}$ que «le monde est tout ce qui advient». Le corollaire de 1.1 est 1.3: «Les faits dans l'espace logique constituent le monde». «L'espace logique» que décrit le Tractatus est donc l'espace dans lequel prennent leur sens toutes les assertions universelles, susceptibles de vérité ou de fausseté, portant sur des «objets quelconques $=\mathbf{x}$ ».

Mais l'espace logique d'un discours réglé ne décrit complètement l'espace assertorique de ce discours que dans le seul cas des logiques formelles. Dans toutes les sciences empiriques, l'espace sémantique dans lequel elles énoncent - appelons-le leur espace assertorique - contient des objets dont la «signification» se trouve nécessairement articulée à des «référents » empiriques: il y a donc toujours lieu de décrire ce que cette forme de «référence» change au sens de leurs définitions d'objets, de leurs raisonnements et de leurs preuves. Au sein même des sciences empiriques, l'espace assertorique des sciences sociales, qui se donnent pour base empirique l'interprétation des interactions humaines observables dans une succession, est encore plus riche en «références» à un contexte historique, c'est-à-dire en « déictiques », «noms propres » et «désignations » de lieux ou temps singuliers - tous «signes» de singularités commandant le sens des assertions et

Ludwig Wittgenstein utilisait par métaphore l'expression de logische Raum pour désigner l'ensemble des contraintes logiques qu'il décrivait dans le Tractatus logico-philosophicus (éd. allemande et trad. anglaise par C. K. Ogden, révisée par l'auteur; préface de Bertrand Russell, Londres, 1922). 
tout autant celui des preuves qu'elles tissent dans une argumentation. Dans les termes qu'employait Frege, une entité nommée dans un langage associe toujours un «sens» ( $\operatorname{sinn}$ ) à une «désignation» (bedeutung), mais cette association ne se manie pas déductivement de la même manière selon la manière de «référer» à laquelle obligent les objets «dénotés ${ }^{3}$. Les règles qui fixent l'utilisation cohérente du rapport entre $\operatorname{sinn}$ (le «sens » d'un terme dans un système linguistique) et bedeutung (la «signification » que lui donne sa référence à l'objet désigné) varient selon les types de discours descriptif, dès lors que les définitions des termes y réfèrent différemment leurs objets: entre les sciences formelles et les sciences empiriques donc, mais aussi entre les sciences expérimentales et les sciences historiques.

En sociologie comme en histoire, le sens des énoncés descriptifs et causaux ne peut être complètement décrit par une sémantique et une grammaire «formelles» susceptibles d'être axiomatisées dans une logique «pure», ni par la seule analyse grammaticale du sens de leurs définitions et principes qu'épuiserait l'axiomatique fermée d'une théorie unifiée dans un «paradigme». Le sens référentiel des discours descriptifs et explicatifs, caractéristiques des sciences historiques, est indissociable d'un «ancrage pragmatique» du sens de tous leurs mots et de toutes leurs assertions dans un contexte particulier d'observation et de description. L'espace assertorique des sciences historiques est donc moins réductible encore à l'espace formel des opérations pures de la logique formelle, que ne l'est l'espace assertorique des sciences hypothético-déductives de la matière ou de la vie qui, capables de mettre en jeu le critère expérimental de la «réfutabilité» des «propositions logiquement universelles », peuvent de ce fait aspirer légitimement au statut de sciences expérimentales.

Marquer cette distance entre sciences historiques et sciences expérimentales n'est évidemment pas «démarquer» les premières de l'ensemble des sciences empiriques, mais seulement des sciences expérimentales. Examiner sur pièces les formes de la description historique et les analyses du sociologue, ne conduit certainement pas à conclure que ces démarches ne relèveraient que de l'approximation ou de la littérature, du reportage subjectif ou d'une libre interprétation des faits. C'est, au contraire, être conduit au constat d'un fait épistémologique: à côté des deux régimes de preuve propres aux sciences formelles et expérimentales, il existe bien un tiers-régime du raisonnement scientifique, doté lui aussi d'un pouvoir d'explication dont on peut décrire le registre d'intelligibilité. Le régime du raisonnement historique combine plusieurs méthodes d'administration de la preuve, et c'est par ce mixage de l'argumentation qu'il diffère constitutivement tant de la déduction logico-mathématique que de l'induction expérimentale, même lorsqu'il en incorpore certains moments. Ce régime se fonde sur un style de pensée qui possède comme les deux autres, ses contraintes et limitations méthodologiques propres, mais aussi ses intelligibilités spécifiques dans l'interprétation scientifique de l'histoire du monde. Pour identifier ce style d'argumentation il faut décrire les ressorts probatoires du raisonnement comparatif, tant dans ses constructions de concepts que dans ses enchaînements d'énoncés.

Gottlob Frege, «Sinn und Bedeutung», in Zeitschrift für Philosophie und philosophische Kritik, $\mathrm{n}^{\circ}$ 100, 1882; «Sens et dénotation» in Ecrits logiques et philosophiques (trad. française de C. Imbert), Paris, Le Seuil, 1971. 


\section{CALCULER, ARGUMENTER, PROUVER}

J'ai dit qu'on pouvait «constater» que la sociologie raisonne dans un espace de la preuve qui est commun à toutes les sciences historiques. Ce constat n'est pas autre chose qu'un constat empirique, accessible au sociologue ou à l'historien qui peuvent facilement l'établir par l'analyse comparative de corpus d'argumentaires prélevés dans l'histoire de leurs disciplines.

Je suis un sociologue d'enquête, ayant travaillé et publié des résultats et des analyses dans divers domaines de la sociologie de l'éducation, de la culture et des arts. Mais, à mesure que je traitais les résultats de ces enquêtes, que je les rapprochais d'enquêtes faites par d'autres, que j'essayais d'interpréter le sens des interactions statistiques entre variables ou des comparaisons entre cas historiques pour en tirer des affirmations qui ne majorent ni ne minorent ce que «voulaient dire» les traitements de données que j'avais montés en systèmes de preuve, je suis devenu perplexe - non pas sceptique, mais de plus en plus perplexe - sur ce je pouvais en affirmer et en écrire légitimement dans mes comptes-rendus: la légitimité scientifique dont les bases m'intriguaient était la légitimité logique et méthodologique du discours des sciences sociales, non, bien sûr, leur légitimité sociale, qui relève de la sociologie de la sociologie. Bref, je me suis interrogé sur le statut épistémologique des démarches de preuve que j'employais, les mêmes que je voyais employer par d'autres sociologues, et qui me posaient les mêmes questions quand je les lisais.

\section{La preuve dans le calcul, l'argumentaire et le récit}

Les épistémologies d'ambition unificatrice, qui ont toujours été légion, ne me persuadaient guère. $\mathrm{Ni}$, malgré le sceau durkheimien qui marque leur origine, les épistémologies qui identifiaient la démarche d'une observation comparative (historique ou statistique) enregistrant des variations concomitantes, à une forme «indirecte» ou «substitutive» de la méthode expérimentale. Ni celles qui assignaient pour idéal méthodologique aux inférences du raisonnement sociologique de s'astreindre à la quantification de toutes ses observations et à la formalisation de tous ses raisonnements, dans l'espoir de parvenir par cette ascèse linguistique à pratiquer enfin de «vraies démonstrations » (dans l'avenir, précise-t-on, pour rendre crédible cette attente du paradis scientifique). Plus éloignées encore du travail mental de l'enquête m'apparaissaient les philosophies de la connaissance qui identifiaient la construction sociologique des concepts à leur élaboration abstraite dans un système philosophique: «l'intuition des essences» loge toujours quelque part dans une philosophie, pour fonder ses évidences dernières.

Popper m'en avait convaincu: une théorie empirique cesse de l'être lorsqu'elle se formule comme une théorie «irréfutable». En perdant sa «vulnérabilité empirique», elle se fait ipso facto «théorie métaphysique». Sur la définition logique d'une «théorie empirique», j'acceptais pleinement l'analyse de Popper: il faut trouver un «critère de démarcation» capable de caractériser de manière univoque le statut logique des énoncés qui peuvent légitimement figurer dans une théorie scientifique. Simplement, pensais-je, s'il faut accepter le modèle logique qui permet à Popper de formuler (dans La logique de la découverte 
scientifique $e^{4}$ ) une définition opératoire de ce qu'est un «test réfutateur» dans le cas des «événements » du monde physique - précisément parce qu'un «événement» est, en ce cas, défini, selon cet auteur, par la répétition de certaines «cooccurrences »- il reste qu'on ne peut suivre Popper dans l'application de ce modèle aux sciences historiques qui, elles, portent toujours sur des aspects des phénomènes dont la pertinence n'est jamais susceptible de répétition à contexte constant ou indifférent. Popper supposait pourtant dans sa polémique contre «l'historicisme» (Misère de l' historicisme $e^{5}$ ) la possibilité d'appliquer son critère de scientificité à toutes les sciences de l'homme. Il plaçait ainsi les sciences sociales dans une impasse en les sommant de choisir entre, d'une part, littérature suggestive ou métaphysique «irréfutable» et, d'autre part, science expérimentale ou calcul des conséquences dans un «modèle». En somme entre une abdication et une illusion. Le gouffre de Charybde et le rocher de Scylla font naufrager également la pertinence des descriptions historiques sur des périls inverses.

L'hypothèse que je voulais, au contraire, tester sur un corpus d'argumentaires sociologiques était qu'on pouvait y trouver des ressorts de l'argumentation qui relevaient pleinement de la preuve empirique, sans qu'ils y prennent pour autant la forme de la «corroboration», telle que la définissait Popper comme absence provisoire de «réfutation». Et qu'ainsi le raisonnement sociologique ne reposait ni sur une explication de la concaténation des phénomènes fondée une «nécessité d' essence »- ce qui reviendrait à majorer métaphysiquement le sens des relations observés ou mesurées entre les phénomènes - ni sur l'établissement d'une relation causale qui reposerait sur de simples «exemplifications» entendues au sens de Popper - ce qui reviendrait à minorer ou annuler la valeur scientifique des inférences - ni non plus, enfin, sur de simples procédés d'éloquence forçant la persuasion du lecteur par des figures de rhétorique. Il m'importait, en effet, d'écarter la troisième interprétation des preuves historiques, à une époque où commençait à se dessiner le linguistic turn qui conduira certains liquidateurs à ne plus voir dans l'argumentation historique qu'une «tropologie» (chez Hayden White par exemple).

Pour sortir de cette auberge où la véridicité du discours des sciences sociales est déformée, par excès ou défaut comme sur un lit de Procuste, il faut tout simplement trouver, dans leur maniement de la preuve, un autre critère de la vulnérabilité empirique que celui de la «réfutabilité» au sens poppérien. On ne peut s'en tenir au dilemme qui condamne à choisir entre la «réfutabilité » d'une hypothèse théorique, capable d'anéantir toute la théorie dont elle dépend en cas de non-corroboration (falsification), et une conception de l'exemplification considérée, dans le modèle poppérien, comme simple «illustration » d'une assertion par un cas bien choisi (ostensive case), laquelle illustration serait toujours d'une valeur probatoire nulle - et cela sans qu'on examine jamais l'inégale sévérité des exigence empiriques propres aux différents modes de l'exemplification ${ }^{6}$. La question devient

K. Popper, La Logique de la découverte scientifique (1959-1968), trad. frçse, Paris, Payot, 1978.

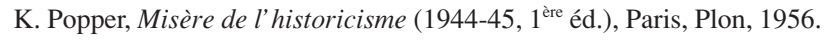

6 Cf. J.-C. Passeron, Le raisonnement sociologique : l'espace non-poppérien du raisonnement naturel, Nathan, Paris, 1991. En particulier dans cet ouvrage, voir la distinction entre le test du « zoo » ou de la «niche écologique » et la prospection au hasard de tous les lieux du monde par le 
donc: par quels schèmes probatoires se trouvent liés, dans un argumentaire, les «raisons» de conclure propres aux sociologues lorsqu'ils répondent par une démarche réglée, mais autrement qu'en apprentis mathématiciens ou en physiciens mimétiques, à la question de la preuve?

\section{Concepts et énoncés dans les sciences historiques}

À la question du régime de la preuve en sociologie, il n'est en effet qu'une réponse pour un sociologue d'enquête: enquêter sur la manière dont raisonnent sociologues et historiens, en comparant leurs raisonnements avec ceux qui se pratiquent dans les différentes sciences sociales. Je me suis donc attaché à constituer un corpus de raisonnements où j'ai fait entrer des textes d'historiens, d'anthropologues, de sociologues, d'économistes, de démographes, de linguistes etc, relevant d'écoles ou d'époques aussi différentes que possible, afin d'examiner comparativement leurs argumentations pour en extraire les ressorts probatoires les plus utilisés ou les plus généraux. Figurent donc dans ce corpus toutes sortes de styles de recherche, des spécimens d'argumentation écrits dans des dialectes différents du langage de la preuve historique; les théories examinées y sont presque toujours concurrentes ou conflictuelles, jamais complètement identiques ni exclusives l'une de l'autre; certaines de ces recherches sont écrites en «langue naturelle» et d'autres dans une «langue artificielle» (statistique par exemple). J'ai voulu en somme décrire empiriquement c'est-à-dire sur pièces, l'espace assertorique du raisonnement des sciences historiques ${ }^{7}$. La spécificité des ressorts probatoires du raisonnement sociologique, telle qu'on peut la décrire en analysant ce corpus, repose ainsi, non sur une définition préalable de la connaissance scientifique ou de la forme théorique qu'elle devrait prendre dans les sciences historiques, mais sur la seule analyse textuelle d'argumentaires scientifiques prélevés dans ces sciences. La spécificité du raisonnement sociologique se manifeste alors aussi bien dans son usage des termes du discours que dans la forme de ses énoncés. En chaque science sociale, le cheminement des preuves est indissociable d'une forme particulière des définitions de mots comme d'un mode particulier de l'enchaînement des inférences.

1). Les déictiques de la nomination quadrillent étroitement le langage et l'argumentation des sciences historiques. Les noms propres et les semi-noms propres, les exemples et les déictiques sont toujours présents dans l'identification des singularités (individuelles ou collectives) sur lesquelles roule leur raisonnement d'imputation causale. Les objets historiques sont identifiés par des termes qui associent toujours la «désignation» à la «description définie»: aucune de ces deux opérations ne suffit, à elle seule, pour fixer leur sens référentiel et leur conférer une fonction probatoire dans l'argumentation. Les concepts de l'historien

zoologue poppérien en quête de cygnes noirs: scolie de la proposition 3.3.1, pp. 388-389. A comparer au raisonnement de Popper sur l'exemplification: La logique de la découverte, op . cit., p. 100 , note 1 .

7 L'explicitation de «l'espace assertorique» des sciences sociales, considérées comme des sciences historiques, est présentée analytiquement dans les «Propositions » qui concluent Le raisonnement sociologique, op. cit., pp. 358-403. 
comme (plus invisiblement) ceux du sociologue reposent à la fois sur des descriptions «partiellement définies» et sur des désignations «multiplement indexées » sur des exemples. De ce fait, si le raisonnement sociologique est souvent probabiliste en ses moments statistiques, s'il peut aussi se prêter à la formalisation de certaines de ses inférences, il ne peut quantifier la probabilité de son interprétation d'ensemble ni en formaliser entièrement l'énonciation. Il reste conjectural dans ses synthèses théoriques, jamais susceptible de se boucler dans un paradigme ou une axiomatique. En revanche, une synthèse socio-historique formulée en «langue naturelle» est toujours susceptible d'être rectifiée par une interprétation documentée qui l'améliore en allongeant une série ou en densifiant une description. L'épistémologie wébérienne avait déjà décrit cette propriété des «concepts » qui ne peuvent, disait-il, fonctionner dans les sciences historiques que comme des «types-idéaux ». Max Weber avait caractérisé comme «stylisation» la construction de tels concepts historiques, toujours tributaires de la comparaison entre «constellations historiques singulières ».

Ainsi définie, la définition idéal-typique apparaît comme radicalement différente de la «définition générique» de forme aristotélicienne, de la définition «génétique» de forme mathématique ou de la «description définie» d'une entité par les logiciens. Il faut cependant admettre que la notion de «stylisation», telle que Weber la mettait au principe de la généralité des concepts sociologiques, prête par sa disponibilité sémantique à une interprétation méthodologique qui risque toujours de disqualifier sa finalité épistémologique. Cette stylisation ne se réduirait-elle pas à une forme faible, à un ersatz de «l'induction», à un procédé douteux de généralisation? Rien d'étonnant si la conception wébérienne de la «typologie» a si souvent été réduite, par des lecteurs cursifs, à l'idée d'une «classification», seulement plus molle et moins exigeante, et la notion de «type idéal » à celle d'un concept approximatif ou flottant. Il me semble que le concept peircéen d' «indexation » rend compte plus précisément que celui de «stylisation » du rapport des mots du sociologue à leur signification.

La définition idéal-typique telle que la pratiquent le sociologue ou l'historien représente bien la forme réglée, parfois strictement codifiée dans une méthode, d'une démarche qui repose sur une opération fondamentale de «l'intelligence naturelle» affrontée à l'appréhension des singularités. Examinons comment on procède quand on essaie d'identifier, dans la communication de tous les jours, une personne qu'on ne peut «présenter». Lorsqu'on ne peut la désigner du doigt, on se guide sur des «désignations » esquissant des types idéaux de contrastes ou de parentés, c'est-à-dire sur des exemples singuliers mis en rapport les uns avec les autres. On dira par exemple: «C'est quelqu'un dans le genre de Marilyn Monroe plutôt que dans le genre de Katharin Hepburn», ou «dans le genre de Lino Ventura plutôt que de Gérard Philippe».

Ce n'est pas là, on le voit, une définition fondée sur une «liste de propriétés » (mensurations, couleur des yeux ou de cheveux etc) qui, si longue soit-elle, n'isolera jamais complètement de toutes les autres l'individualité visée. Sauf évidemment, lorsque l'on peut remplacer la liste des propriétés d'un individu par une photographie expérimentalement infaillible de son individualité, comme dans le cas des empreintes digitales, ou mieux des «empreintes génétiques ». Dans ce cas, remarquons-le, l'identification certaine d'une singularité joue bien un rôle opératoire s'il s'agit par exemple de prouver (comme dans l'enquête policière) la pré- 
sence de tel ou tel acteur dans une interaction. Mais elle n'ouvre pas, par l'information qu'elle transmet, la possibilité de comparer les effets d'autres caractéristiques des acteurs dans d'autres interactions. Tant qu'elle reste autosuffisante l'identification d'une singularité n'est pas un «embrayeur» de la connaissance comparative. En revanche, l'identification qui repose sur des analogies et des séries ouvre d'autant plus d'entrées au raisonnement comparatif que la liste des propriétés reposant sur des indexations différentes est plus longue. La définition qui intègre des moyens analogiques d'identification permet, si celui à qui on la communique connaît déjà les «comparants» et si la liste en est bien articulée, d'identifier plus de « comparés » c'est-à-dire de les connaître sous plus de rapports différents par le même concept typologique. Dans les interactions et les interlocutions quotidiennes, l'identification d'une individualité s'opère bien par une «indexation» de la généralité sur des exemples singuliers, en même temps que sur des contrastes ou des analogies entre ces exemples, plus ou moins longuement décrits aux fins d'analyse. En son principe logique, la démarche est encore la même pour la désignation d'une singularité dans le discours de l'historien. Celuici est seulement contraint à des descriptions de «cas» plus longues ou plus travaillés, puisque la désignation du «référent» d'un concept porte alors sur des «configurations historiques globales», c'est-à-dire sur des singularités descriptibles sous plus de rapports différents ${ }^{8}$.

Les concepts de la description historique reposent sur une opération de «logique naturelle » ${ }^{9}$ qui précise l'objet qu'elle vise par référence à des singularités de lieux et de moments. Je les appelle des semi-noms propres. Ils ne sont pas de «purs» noms propres puisque l'indexation sur des singularités s'y trouve toujours associée à la définition par énumération de propriétés. Pour revêtir tout son sens descriptif et fonctionner dans une argumentation, une conceptualisation idéal-typique associe le langage de l'analyse de «traits » à celui de la désignation de «cas». L'historien qui utilise, par exemple, le concept de «féodalité» doit, pour être compris de l'historien auquel il s'adresse, avoir en commun avec lui la définition de quelques propriétés génériques (confiscation locale du produit du travail agricole, monopolisation des moyens de guerre et des arsenaux, pouvoir

8 L'originalité sémantique de la deixis - c'est-à-dire de l'acte par lequel on « désigne du doigt » une individualité à défaut de pouvoir l'appréhender par une «description définie » - avait depuis longtemps tourmenté les grammairiens, confrontés à l'impossibilité de donner une définition non-tautologique du sens des «noms propres». Dans sa sémiotique, Peirce avait défini «l'index» (à côté du «signe» et du «symbole») comme une catégorie spécifique de signifiant: l'index « dirige l'attention vers son objet par une compulsion aveugle (blind compulsion) ». La question de la définition des déictiques est devenue centrale chez les philosophes et les logiciens du langage depuis l'article de K. Donellan: «Reference and Description», The Philosophical Review, LXXV, 1966. Cf. la place de la désignation des singularités dans un système logique comme celui de Kripke, «Naming and Necessity» in Semanticcs of Natural Languages, Davidson \& Hartman eds, 1972. Cf. surtout E. Benveniste, Problèmes de linguistique générale (2 tomes), Paris, Gallimard (1966, 1974). Et sur «l'ancrage pragmatique» de tout nom propre ou déictique, G.-G. Granger: «A quoi servent les noms propres?», numéro spécial sur «Le nom propre», J. Molino (sous la dir. de), Langages, Paris, 1982 .

9 Au sens où J.-B Grize en a développé la description: Logique et langage, Gap, Ophrys, 1990; Logique moderne, Paris, Mouton, 1969, 1973. Cf. du même auteur la définition de la «schématisation» in «Les discours du savoir: pour un dialogue avec Jean-Claude Gardin», Revue européenne des sciences sociales, XXXVIII, 2000, pp. 131-138. 
hiérarchisé selon une dépendance personnelle, renforcement symbolique de la hiérarchie du vasselage par un code d'honneur militaire etc). Mais il doit se référer en même temps, pour interroger les contextes, à la connaissance qu'il a existé des cas historiques de féodalité, descriptibles mais inépuisables à une analyse exhaustive: Chine des Royaumes Combattants, Japon de Kamakura, Chrétienté médiévale etc. Il en va de même pour tout concept doté de généralité explicative, où le rôle des coordonnées spatio-temporelles et celui des propriétés trans-historiques, également indispensables, ne sont pas également visibles dans la caractérisation des faits: "césaro-papisme», «bonapartisme», «fascisme», «stalinisme» semblent des concepts plus historiques que «ville marchande», «légitimité charismatique», «capitalisme», «monachisme» ou «secte», mais ils ont la même texture sémantique, que j'appelle ici celle du «semi-nom propre». De même encore pour leurs particularisations: capitalisme moderne ou antique, aventurier, marchand ou financier, rhénan, anglo-saxon ou japonais, dans lesquels seule la désignation des cas permet d'isoler et d'articuler des propriétés différentielles par la comparaison de «constellations globales». Les «cas» historiques ne sont pas de simples «exemples» qu'on pourrait oublier, une fois formulée par induction la définition générique du concept par une énumération de propriétés universelles, nécessaires et suffisantes.

2). Dans l'administration d'une preuve historique interviennent sans cesse comme opérateurs des schèmes de raisonnement qui sont irréductibles aussi bien (a) aux «opérations » que définit une logique formelle que (b) aux «figures » que décrit une rhétorique. On peut prendre comme exemple de schème celui de l'adversation: «Certes ... mais» ( c'est celui qui a la plus haute fréquence dans mon corpus). Un «schème» de cette forme assertorique ( Certes $\mathbf{p}$ est vrai, mais $\mathbf{q}$ l'est aussi»), peut se présenter selon les argumentations sous une multiplicité de formes grammaticales, mais, dans sa fonction argumentative, il fonctionne toujours comme un «embrayeur» de la preuve. Sa valeur probatoire n'est pas invariante, comme le serait celle d'une opération logique. Elle est fonction des nouvelles descriptions auxquelles il introduit en obligeant, par sa forme potentiellement interrogative, à poursuivre le raisonnement. Sa force probatoire est, par exemple, de valeur nulle lorsque le schème fonctionne comme une précaution oratoire qui se suffit à elle-même, parfois même comme un habillage dissimulant une contradiction pure et simple: «S'il est vrai que p... il est vrai aussi que q (non-p)» disent de nombreuses phrases adversatives, où q ne signifie finalement rien de plus que la contradictoire de $\mathbf{p}$ dans le contexte de l'argumentation. Ce même schème de l'adversation peut au contraire fournir le ressort heuristique d'une preuve par analogie, contraste ou spécification, lorsqu'il oblige le chercheur à rechercher d'autres constats historiques ou d'autres mesures susceptibles de faire comprendre la relation entre la vérité de $\mathbf{p}$ et la vérité de $\mathbf{q}$, de la «justifier» en enrichissant la connaissance du contexte dans lequel prennent un sens compatible les deux constats qui se présentaient comme antithétiques au départ de l'argumentation. L' «adversation» de deux énoncés est plus riche de conséquences potentielles que la «conjonction» de deux propositions, qui n'engendre en logique formelle que ce que peut en dire, une fois pour toutes, sa «table de vérité »: la proposition complexe $\mathbf{p}+\mathbf{q}$ est vraie si et seulement si chacune des deux propositions élémentaires qu'elle connecte est vraie. 
Il en va de même pour les schèmes analogiques de description lorsqu'il sont utilisés afin d'augmenter l'intelligibilité d'une comparaison entre «cas», par exemple: $\mathbf{a}$ est à $\mathbf{b}$ comme $\mathbf{c}$ est à $\mathbf{d}$, comme $\mathbf{e}$ est à $\mathbf{f}$ et comme $\mathbf{g}$ est à $\mathbf{h}$....etc. ${ }^{10}$ Lorsque je lis chez un historien que le Synode de Dordrecht est au Concile de Trente comme, dans ce synode de la Réforme, la théologie calviniste de la prédestination est à l'arminianisme des «remonstrants» ou des gomariens, et comme, dans la Contre-réforme tridentine, la théologie jésuite du libre-arbitre est à la théorie janséniste de la grâce, ou comme, dès l'Antiquité, la théologie du salut chez St Augustin est à celle de Pélage, ma «compréhension» de l'histoire des doctrines ne tire de ce schème d'intelligibilité analogique une amélioration de l'explication des idées et des événements religieux que si celui-ci fait mieux ressortir la signification de récits et de descriptions historiques en les structurant sous cette forme synoptique. J'ai remarqué sur mon corpus d'argumentaires que la fréquence des schèmes analogiques d'organisation de la preuve était d'autant plus élevée, leur intrication d'autant plus serrée dans la «compréhension» d'un concept et leur rôle probatoire d'autant plus important qu'on les trouvait dans des textes où l'établissement de la preuve reposait plus exclusivement sur la densité des comparaisons historiques: Weber, Dumézil ou Braudel ${ }^{11}$. La reconstruction frégéenne de la logique sur la base du «principe d'extensionalité » ne rend pas compte de l'usage que les sciences historiques font de leurs concepts. Sociologie historique, anthropologie comparatiste ou histoire comparée ne désignent finalement que des mises en œuvre, différemment conformées selon différentes traditions disciplinaires, d'une même logique de la preuve qui opère en tout raisonnement sociologique. Un schème prouve quelque chose par l'enrichissement des descriptions empiriques auquel il oblige pour éclaircir sa portée, pour la diversifier ou la limiter. L'acte logique de «faire preuve» par lequel un schème «montre» le sens d'une relation de causalité en l'interprétant est, dans une science historique, logiquement irréductible à l'acte de «démontrer» tel qu'il s'exprime dans une déduction. Mais il l'est tout autant au simple constat factuel qu'une figure du discours à réussi à «persuader» un public.

3). Par voie de conséquence, la référence à un contexte historique, minutieusement analysé ou allusivement désigné, est toujours présente, aussi bien dans les termes descriptifs que dans les énoncés explicatifs d'un raisonnement sociologique. Dans une science historique qui, par définition, ne connaît pas de répétition des phénomènes se déroulant à contexte constant ou indifférent, les preuves sont toujours tributaires d'un contexte singulier d'observation, de mesure et d'argumentation. La multiplicité concurrente des théories comme la forme «idéal-

10 Cf. sur l'usage de ce schème chez Dumézil par exemple, Le raisonnement sociologique... op. cit., pp. 384-385.

11 Chez Max Weber, le texte est celui de la conclusion de Konfuzianismus und Taoismus, in Gesammelte Aufsätze zur Religionssoziologie (Tübingen, Mohr, 1920-1921), trad. frçse, Sociologie des religions, Paris, Gallimard, «Résultat: confucianisme et puritanisme», pp. 379-409.; chez Dumézil: Les dieux des Indo-européens, Paris, PUF, 1952; chez Braudel le résumé qu'il donne de sa théorie des économies-mondes (Civilisation matérielle, Economie, Capitalisme: $X V^{e}-X V I I I^{e}$ siècle, Paris, A. Colin, 1979) dans trois conférences données à l'Université John Hopkins: La dynamique du capitalisme, Paris, Arthaud, 1985. 
typique » des concepts historiques, l'une et l'autre liées à la variabilité intrinsèque des principes de description du cours du monde historique, alimente depuis toujours un vain combat épistémologique entre ceux qui veulent à tout prix réduire cette anomie théorique (que ce soit par l'éclectisme ou par la re-fondation) et ceux qui l'interprètent comme le signe d'une définitive disqualification scientifique des sciences sociales. C'est en vain qu'on déplore le foisonnement et l'entrecroisement des théories sociologiques: la cohabitation conflictuelle des langages de description du monde est constitutive de l'espace de la preuve au sein lequel raisonnent (pour le meilleur ou pour le pire) les sciences historiques. La diversité de leurs syntaxes et de leurs lexiques d'argumentation n'exprime qu'un dosage différent des méthodes descriptives, narratives ou quantitatives; l'effet probatoire de ces méthodes revêt la même forme logique dès qu'on les applique à une comparaison entre des «cas» dont la signification historique exige cette logique argumentative, parce que le maniement d'une telle signification est inaccessible aux règles et définitions d'un calcul au sens strict.

De là aussi, me semble-t-il, lorsque nos disciplines sont sollicitées par un décideur en vue d'applications ou d'orientations pratiques, l'inconfort avéré des chercheurs qui pratiquent une science historique. Leur position est en effet fort différente de celle des sciences de la «loi» et du «modèle», en ce qui concerne la prévision ou le pronostic, l'expertise politique ou le conseil efficace. Derrière leur hésitation à conseiller sans ambages le prince ou la firme, comme le font volontiers l'économiste ou le démographe - exemptés de toute circonstanciation de leurs constats par l'intemporalité de leurs modèles - se manifeste en effet une tout autre organisation de l'établissement des rapports entre la détermination des effets par des causes antécédentes et l'interprétation contextualisée du sens culturel des actions et interactions. Le sens culturel d'un acte ne peut être allégué que comme un sens plausiblement intentionnel, et la probabilité de cette allégation ne peut être prouvée empiriquement que dans un contexte ou un récit.

\section{III. «FAIRE PREUVE» DANS LES SCIENCES DE L'INTERPRÉTATION}

Les sciences historiques ne peuvent formuler leur tâche explicative sans découvrir qu'elle implique un acte d'interprétation. Comme le formulait Max Weber: «C'est une chose que de comprendre ce que dit quelqu'un; et autre chose de comprendre les raisons pour lesquelles il le dit». Comprendre sociologiquement, c'est comprendre les deux choses en établissant un lien entre elles.

\section{Interprétation et causalité}

Dans une science historique qui porte par définition sur des interactions sociales se déroulant dans le temps, l'intelligibilité de l'explication suppose l'interprétation des actes, c'est-à-dire la reconstruction (plus ou moins probante) de leur sens intentionnel par référence aux valeurs de la culture où on les observe. Je ne dirais certainement pas que les explications des sciences physiques sont des constats aveugles que l'expérimentation imposerait au physicien sans qu'il y «comprenne» rien. Elles reposent elles aussi sur des «interprétations». Mais il 
existe une différence fondamentale entre l'intelligibilité d'une «action» lorsqu'on la comprend par ses motifs et l'intelligibilité d'une «loi» lorsqu'on la comprend en sa nécessité, c'est-à-dire l'intelligibilité que procure à l'interprétation de la causalité une théorie nomologique inscriptible dans un paradigme explicatif. L'universalité d'une assertion se comprend toujours de la même manière, puisqu'il n'y a rien d'autre à comprendre dans une loi physique ou une nécessité logique que l'idée de sa validité universelle. Une fois qu'on l'a établie déductivement ou expérimentalement, l'universalité d'un théorème ou d'une loi implique ipso facto l'explication complète de la série infinie des singularités qu'elle subsume, puisque la considération du détail des «cas» n'y ajouterait rien.

Tout au contraire, l'unité conceptuelle propre à la démarche interprétative appliquée à des actions ne s'exprime ni ne s'épuise dans l'unification formalisée de ses principes: elle réussirait sinon à se formuler comme une «axiomatique». Elle ne se réalise pas non plus dans l'inscription de toutes les recherches au sein d'une théorie unique, formulable dans un langage unifié: ce serait alors un «paradigme» au sens de Kuhn. Une telle «pureté» des éléments sur lesquels roule de bout en bout un raisonnement ne s'observe dans aucune des sciences sociales, sauf, bien sûr, lorsqu'elles choisissent d'autonomiser, par décision de méthode, un objet qu'elles peuvent ainsi définir a priori sans référence à un contexte historique: par exemple la transaction économique, lorsqu'on la définit comme un système de relations entre des «prix» et des «quantités» afin de construire mathématiquement une théorie «pure» du marché, comme l'est la théorie de l'équilibre entre l'offre et la demande de Walras-Pareto.

Autrement dit, dans les sciences de l'observation contextualisée, l'étalon de la scientificité est double. L'intelligibilité de l'explication réside dans l'interdépendance entre deux caractéristiques du langage de description du monde historique: d'une part la consistance sémantique des argumentaires qui lient les assertions dans un discours et, d'autre part, la vulnérabilité empirique des assertions de l'argumentaire lorsqu'on les formule dans des protocoles d'enquête: le sens probatoire des protocoles dépend ici de descriptions dont la trame reste toujours celle d'un récit. La deuxième exigence distingue l'herméneutique sociologique de l'herméneutique philosophique fondée sur la «variation imaginaire » c'est-à-dire, au bout du compte, sur l'«intuition des essences » au sens de la phénoménologie husserlienne. Dans une science historique, un langage théorique n'est scientifiquement explicatif que s'il permet d'énoncer ce que Max Weber appelait une «chance typique» de co-occurrence ou de succession d'événements, c'est-à-dire une interprétation plausible de leur association (certaine ou probable) menant d'une description empirique à une autre. Lorsqu'on constate qu'un raisonnement de causalité fait ainsi intervenir des schèmes d'argumentation dont la force de preuve dépend de protocoles de confirmation historique de l'interprétation, alors - alors seulement, mais alors certainement - une telle forme d'inférence définit un registre spécifique de la preuve, autrement dit, un «style» du raisonnement scientifique $^{12}$.

12 Au sens de A.C. Crombie qui a identifié et minutieusement décrit, sur l'histoire longue des sciences occidentales, six «styles de la pensée scientifique»: Styles of Scientific Thinking in the European Tradition: the History of Argument and Explanation especially in the Mathematical and Biomedical Sciences and Arts, 3 vol, London, Duckworth \& Co, 1994. 
L'analyse de mon corpus d'argumentaires sociologiques m'a ainsi conduit à définir un «style» de preuve, que j'appelle le style du «raisonnement sociologique». En le nommant «sociologique», je ne privilégie en rien la sociologie, je ne fais qu'économiser les qualificatifs. C'est, en effet, le même que l'on retrouve à l'œuvre, à des degrés divers de composition avec d'autres styles de la preuve, dans toutes les sciences sociales. Me référant aux six styles de la pensée scientifique (styles of scientific thinking) qu'avait distingués Alistair Crombie ${ }^{12}$, je crois le style du raisonnement sociologique assez différent de ces styles classiques pour le caractériser comme un «septième style ${ }^{13}$. C'est un style qui associe dans un argumentaire composite des méthodes d'inférence de nature logiquement hétérogène, inscrites dans des «univers du discours » non entièrement superposables, mais capables d'améliorer par cette association leurs forces probatoires au service d'une explication empirique.

Les contraintes qui définissent cet univers de la preuve commandent quatre conséquences :

1). D'abord la pluralité des théories, qui découle nécessairement de la pluralité des langages de description applicables au monde historique: le cours du monde historique exclut la possibilité d'une induction fondée sur l'observation d'une répétition stricte des relations pertinentes. Il faut accepter le fait de cette cohabitation entre théories concurrentes, mais il faut aussi en tirer toutes les conséquences dans le débat entre chercheurs et le maniement de l'objection ou de l'accord? Quel sens prend la vérité des assertions explicatives dans un univers d'intelligibilités disjointes ${ }^{14}$ ? Quand la «véridicité» de l'explication historique ne repose plus exclusivement ni sur la nécessité logique de la déduction ni sur une théorie «falsifiable» au sens poppérien, la force d'une analyse sociologique que les chercheurs ressentent pourtant comme «plus» ou «moins» probante ne départage pas les théories comme le «tout ou rien» de la «réfutation» définitive ou de la «corroboration» provisoire dans une science expérimentale. Les ressorts probatoires de cette véridicité permettent cependant de classer les théories sociologiques en fonction d'un double critère: celui de la fécondité interprétative de leur langage et celui de leur aptitude à schématiser des protocoles d'enquête. C'est là un double classement de l'utilité scientifique des théories concurrentes que chaque chercheur pratique quotidiennement dans et par l'utilisation qu'il en fait. Chacun des deux classements est lui-même variable selon les questions que pose un chantier empirique déterminé et selon l'état du débat théorique entre chercheurs à un moment donné de l'histoire de la discipline. Mais «pluralité théorique» ne signifie ni une absence de hiérarchie entre l'utilité conjoncturelle de chacune des théories, ni une anarchie sémantique qui les condamnerait toutes au non-sens, Sauf, bien sûr, aux yeux du théoricien scientiste qui ne peut reconnaître la théorie que sous la forme d'un paradigme excluant les autres; ou pour le philosophe qui pense pouvoir caractériser la vérité d'une théorie, hors de tout chantier ou contexte.

13 J.-C Passeron, « Logique et schématique dans l'argumentation des sciences sociales », Revue européenne des sciences sociales, Genève, Droz, n 107, 1997, pp. 176-178.

14 J.-C. Passeron, « De la pluralité théorique en sociologie: théorie de la connaissance sociologique et théories sociologique», Revue européenne des sciences sociales, n 99, 1994, pp. 71-116. 
2). Le caractère «idéal-typique» des concepts et des assertions historiques qui constituait le socle de l'épistémologie de Max Weber est souvent malmené par les commentateurs. Il importe seulement de voir que cette épistémologie conduit à une méthode de construction des définitions qui garde l'avantage de dissiper un malentendu essentiel et toujours renaissant sur l'objet dont parlent les sciences historiques. Lorsque la nature logique des définitions sociologiques est mal identifiée, la confusion avec d'autres formes de définition engendre de perpétuels contre sens sur ce que prouve une argumentation sociologique. Dans une science historique, les concepts descriptifs sont toujours construits (que le chercheur l'aperçoive ou non) par «stylisation » et non per genus proximum et differentiam specificam, c'est-à-dire par énumération de propriétés comme dans une classification. J'ai seulement voulu préciser la description épistémologique qu'avait menée Max Weber qui, en scrutant logiquement l'usage que la sociologie fait du langage de l'identité et du devenir des objets historiques, avait montré le rôle central des concepts idéal-typiques en toute méthodologie historique: ceux-ci constituent l'outil indispensable à une exploration des «singularités » par le raisonnement comparatif, en concentrant dans une typologie les acquis descriptifs des comparaisons passées. J'ai voulu montrer, en analysant la fonction des «déictiques » dans l'argumentation sociologique, le rôle que jouent les «coordonnées spatio-temporelles » d'une événementialité historique jusque dans le langage le plus abstrait du sociologue. En tous les mots qu'il emploie, répétait Weber, le sociologue, comme l'historien ou l'ethnologue, parle par concepts et énoncés idéal-typiques toujours indexés sur des séries de cas, c'est-à-dire, comme je le paraphrase ici, par semi-noms propres et énoncés assortis de déictiques.

La singularité des « référents » qui donne leur sens au discours comparatif des sciences sociales ne se manifeste pas de la même manière en chacune. Elle s'aperçoit, par exemple, plus facilement dans la texture logique des concepts du sociologue que dans la forme de ses énoncés: aspirant à un maximum de généralité, ceux-ci semblent souvent se confondre avec la formulation d'une loi, dont la validité universelle serait seulement atténuée en se formulant comme une «régularité » sociologique. A l'inverse, la référence à la singularité des «cas » s'aperçoit plus facilement dans les énoncés de l'historien, toujours coulés dans l'événementialité d'un récit, que dans ses concepts généraux: affichant la neutralité des noms communs venus du langage courant, ceux-ci paraissent être suffisamment définis, quel que soit le cas décrit, par quelques propriétés de portée trans-historique. C'est ne pas voir que les concepts de l'historien sont toujours, par l'indexation du sens qu'ils prennent dans un récit, les résultats concentrés d'une comparaison antérieure entre des cas, comparaison implicite où s'est conservé quelque chose de la singularité de chacun; et que les assertions du sociologue restent indexées historiquement sur une série comparative de cas, en dépit de la syntaxe du discours généralisant qui les formule abstraitement comme des régularités,

3). Cette texture logique des termes et des propositions fait apparaître la contextualisation des explications comme un caractère constitutif de toutes les sciences historiques. Un contexte historique est toujours singulier et donc inépuisable à une description finie. Les propriétés qui, dans un contexte donné, sont pertinentes pour une explication ne peuvent être épuisées une fois pour toutes par une liste finie de « variables » et de leurs valeurs. L'explication sociologique reste ainsi 
toujours ouverte à de nouvelles enquêtes, construites sur de nouvelles descriptions organisées par de nouveaux concepts. On peut toujours améliorer ce qui, dans le maniement de la méthode comparative, distingue de l'analogie fondée sur une série trop courte ou sur un apparentement «sauvage» le contrôle plus détaillé de la «parenté» des contextes. Et les types idéaux de contextes ne peuvent être construits autrement que les types idéaux de processus particuliers: plus le concept vise à nommer en sa généralité idéal-typique une configuration globale, plus exigeante se trouve être son indexation historique sur une série plus longue d'oppositions ou de ressemblances entre cas différents. La généralité d'un typeidéal repose sur une démarche inverse de celle de la généralisation par induction, qui anonymise en les répétant les expériences quelle agrège.

4). La démarche interprétative est indissociable de la démarche explicative dans les sciences de l'action et de l'interaction sociales, dès qu'on les définit comme des sciences historiques. Cela Weber l'avait posé avec force dans sa théorie des rapports entre «compréhension» et «interprétation»: la seconde ne se réduit pas à la première ${ }^{15}$. La ré-interprétation du sens culturel s'opère dans les détours d'un raisonnement laborieux qui dote les interactions sociales d'un «sens supposé » en le reconstruisant sur des analogies et des contrastes. La redéfinition réciproque des cas singuliers renforce ou rectifie la preuve d'une imputation causale à mesure que s'allonge la chaîne comparative des descriptions ou des mesures. Les sociologues recourent tous, à un moment ou un autre, au travers d'une méthode ou d'une autre, à l'interprétation pour expliquer une succession d'actions et d'effets ou une interaction entre variables.

L'objectivité ostentatoire du langage de la description ou l'appareillage statistique du traitement de données dissimule facilement, dans un discours sociologique, le rôle sémantique des actes d'interprétation. Le sociologue qui ne veut pas être suspecté d'herméneutique «subjective» laisse volontiers entendre par sa terminologie que les instruments logiques ou statistiques de ses interprétations sont les seuls garants d'une interprétation présentée comme «objective» parce que supposée soumise aux seules règles de l'automatisme des instruments. L'interprétation du sens des actions qui fonde l'intelligibilité des relations causales répugne souvent à s'avouer comme telle. Songeons par exemple à la place presque invisible de l'interprétation dans le Suicide de Durkheim, où s'est fondé le mariage méthodologique de la sociologie avec le traitement statistique des «variations concomitantes ». L'analyse du texte qui a imposé et impose encore aujourd'hui l'ouvrage comme un argumentaire exemplaire de ce qu'est une administration de preuves dans une démarche de sociologie quantitative montre pourtant, au fil des arguments, le rôle actif que joue l'interprétation du «sens» des actions dans la construction par Durkheim des trois «types de suicide». Les types durkheimiens du suicide sont des «types idéaux » qui construisent la consistance sémantique du

15 M. Weber, Wirtschaft und Gesellschaft, Tübingen, J.C.B. Mohr (1 $1^{\text {ère }}$ éd., 1922; $4^{\text {ème }}$ éd. par J. Winckelmann, 1956); trad. frçse partielle, Economie et société, Paris, Plon, 1971. Cf. «Les concepts fondamentaux de la sociologie», pp. 3-59 et, plus particulièrement, les «Fondements méthodologiques », pp. 4-19. Max Weber avait approfondi et détaillé les formes et les niveaux de l'interprétation historique dans un long article (non traduit en français): «Rosher und Kniess und die logischen Probleme der historishen Nationalökonomie » (1903-1906). 
type social, en lui attribuant un sens plausible, en même temps qu'ils mettent cette consistance à l'épreuve d'une série de variations statistiques et de comparaisons historiques argumentées: le ressort de la preuve repose bien sur la congruence entre le sens culturel des conduites et le sens des corrélations observées, entre variations des taux de suicide et variations des valeurs d'une série de variables. Réduits à la valeur numérique de leur seule signification statistique ou interprétées en «compréhension » naïve, ces corrélations, contrastes ou parallélismes resteraient incompréhensibles ou psychologiquement contradictoires. Rendre plausible une interprétation du sens des interactions sociales par le recours à la comparaison historique constitue toujours un renforcement de la probabilité causale établie par l'enquête synchronique ou le récit singulier. Et vice versa.

Ces caractéristiques de l'argumentation valent-elles globalement pour toutes les sciences sociales? Oui, parce que celles-ci ne sont sociales que pour autant qu'elles restent des sciences historiques. À condition d'ajouter, cependant, que toutes les sciences sociales ne sont pas des sciences historiques au même degré. Certaines parviennent, par les choix de leur construction d'objet, à suspendre une ou plusieurs des quatre contraintes que je viens d'énumérer; mais c'est toujours au prix d'un oubli (plus ou moins complet, plus ou moins momentané) du caractère historique de leur objet. L'analyse de la preuve que je présente ici s'entend donc des sciences sociales les plus synthétiques: sociologie, histoire, anthropologie (ou ethnologie). La question du «style» de la preuve se présenterait différemment dans les sciences sociales dites «particulières» qui s'attachent prioritairement à la construction de «modèles» ou à l'élaboration de «théories pures»: économie mathématique, théorie des jeux, démographie, linguistique, sémiologie. En fait, pour caractériser la démarche qui leur permet de construire des modèles et des théories pures, il vaudrait mieux les qualifier de sciences autonomisantes: elles autonomisent, en effet, leurs définitions de toute référence à un contexte historique particulier. Elles se trouvent ainsi placées dans une situation méthodologique qui participe à la fois des sciences du calcul logico-mathématique et des sciences de l'explication historique: leurs théories oscillent souvent entre ces deux styles, ou les alternent; il arrive qu'elles les confondent comme s'il s'agissait du même. Le débat entre les sciences synthétiques et les sciences autonomisantes s'obscurcit dès qu'elles affrontent leurs intelligibilités distinctes en supposant identique l'espace logique de leurs actes de probation.

Si on identifie ainsi plusieurs formes, irréductibles les unes aux autres, d'administration de la preuve dans la démarche des différentes sciences, on doit au moins distinguer radicalement, trois grands «régimes de scientificité », c'est-àdire trois logiques distinctes de l'intelligibilité scientifique susceptible d'être construite dans le cadre d'un raisonnement réglé: (1) le régime logico-formel, (2) le régime expérimental et (3) le régime contextuel ${ }^{16}$. Il faut alors tirer les conséquences qu'impose à l'argumentation une définition cohérente de «l'espace logique » des assertions scientifiques dans ces trois mondes de «faits». C'est cette clarification minimale qui est allégrement méconnue dans la définition scientiste de l'intelligibilité sociologique (en particulier celle de la sociologie quantitative), lorsqu'on continue à la présenter comme si elle était issue d'une induction fondée

16 Le raisonnement sociologique, op. cit; en particulier: «Propositions», pp. 363-395. 
sur l'expérimentation ou, comme on le dit aujourd'hui par un euphémisme ambigu, sur une «quasi-expérimentation».

Afin d'éviter de confondre ainsi - par un sous-entendu générateur de confusion et de polémiques dans l'évaluation réciproque de leurs preuves par les chercheurs - l'espace assertorique propre aux sciences sociales avec celui des sciences formelles ou expérimentales, j'ai voulu, au contraire, montrer à la fois la spécificité, l'unité et la signification de l'intelligibilité historique que vise toute science sociale, quelle que soit la diversité de ses techniques d'analyse et des formes discursives de confrontation entre calcul théorique et observation empirique qu'elle pratique. Lorsque, dans une science sociale, nous «comprenons » un ensemble de faits ou de relations à travers le langage particulier de la théorie empirique qui les met en rapports de signification et de causalité, c'est toujours à travers un réseau sémantique de concepts issus de la comparaison historique, à ce titre jamais complètement dissociables d'un «contexte singulier» de description. Les concepts descriptifs du sociologue restent, en effet, en tant que concepts comparatifs, indissociables de descriptions de «cas» menées dans des contextes susceptibles d'être «apparentés» ou «contrastés» au sein d'une série comparative, mais jamais d'être traités comme identiques ou différents «toutes choses égales par ailleurs $\gg{ }^{17}$. Il n'y a pas d'autre définition possible des sciences sociales que par l'identification de la part de raisonnement comparatif que contient chacune en tant que science historique; et pas d'autre définition du raisonnement sociologique que par la forme d'argumentation conjecturale, toujours améliorable, que lui impose la description d'actions dont le sens n'est adéquatement décrit que si on le singularise dans un contexte culturel, inépuisable à la «description définie» des logiciens, mais susceptible de descriptions idéal-typiques par un historien.

\section{MODÈLES PURS ET MODÈLES À DÉICTIQUES}

Cette analyse conduit à distinguer le statut épistémologique des «modèles universels » et celui des «modèles à déictiques ${ }^{18}$. Les premiers sont exempts de tous «noms propres» (de toute nomination de singularité). Dans les sciences empiriques qui sont capables d'expérimenter, la preuve repose, en effet, sur la possibilité de répéter des expériences menées « toutes choses égales par ailleurs ». On dispose ainsi, lorsqu'on peut recourir à l'expérimentation au sens plein, d'une forme forte de la vulnérabilité empirique. «Forte» veut dire qu'on peut décrire sa méthode en des termes exclusivement logiques, susceptibles d'être universalisés, quel que soit le matériau singulier de la recherche, quelles que soient les coordonnées spatio-temporelles des faits sur lesquels on raisonne. Karl Popper a construit le modèle logique d'un tel raisonnement expérimental, en subordonnant sa force probatoire à l'impératif de «réfutabilité »: un seul «énoncé existentiel singulier» suffira toujours à réfuter une «proposition logiquement universelle». Exemple simple: la deuxième loi de la Thermodynamique (celle de la dégradation de

17 Le raisonnement sociologique, op. cit. plus particulièrement, pp. 377-385

18 J.-C Passeron, «Le modèle, l'enquête et le récit», in N. Mayer, (sous dir. de), Les modèles explicatifs du vote, Paris, L'Harmattann, 1997, pp. 28-42. 
l'énergie). Il y aurait réfutation définitive de la théorie dans son ensemble si, un jour, en un point du monde, on pouvait observer qu'il existe une «machine à mouvement perpétuel». En revanche, dans les modèles du second type, c'est une observation, toujours extensible à de nouveaux «cas » indissociables de leurs coordonnées spatio-temporelles, c'est-à-dire l'usage du raisonnement sociologique, qui garantit le fondement empirique de l'explication. Le sens historique qui fournit son contenu aux affirmations d'un raisonnement sociologique se perd dès que l'on désassortit de telles affirmations de leurs coordonnées spatio-temporelles.

On peut facilement exemplifier la différence logique entre les deux familles de modèles en comparant un modèle "pur» d'économiste et un modèle «à déictiques» d'historien de l'économie. Le modèle de Hotelling, par exemple, démontre mathématiquement que sur un territoire quelconque des firmes qui se trouvent en concurrence pour offrir des biens ou des services aux consommateurs résidant dans cet espace ont toujours intérêt, pour maximiser leurs ventes, à adopter une stratégie rationnelle de concentration de leurs points d'offre au centre du territoire (à égale distance des deux extrémités d'un segment de droite symbolisant l'espace des transactions). La démonstration établit une conséquence de valeur universelle, exempte de spécifications en ce qui concerne la nature du «bien» ou du «gain» pour les acteurs de la transaction: rien d'étonnant alors si le modèle a été immédiatement adopté et transposé par les politologues pour expliquer «la course vers le centre» des programmes politiques et des stratégies électorales $^{19}$. Le modèle de Keith Hopkins, historien de l'économie antique, vise au contraire à expliquer le déroulement d'une histoire singulière, celle de l'inversion des courants commerciaux entre l'Italie et les provinces de l'Empire au cours de l'histoire de Rome. Le modèle est construit sur des axiomes et des hypothèses théoriques concernant l'impôt et les effets commerciaux de son utilisation. Mais le raisonnement qui conduit dans la logique de ce modèle à des vérifications historiques doit, pour être probant, particulariser immédiatement les propositions théoriques de départ en décrivant leurs effets dans un contexte historique et cartographique singulier, celui de l'évolution des transits, des stationnements militaires et des statuts provinciaux dans l'espace politique des conquêtes de la République puis de l'Empire romains, de -200 à +400 , en distinguant trois zones économiques qui différent par l'importation ou l'exportation de l'impôt, par le développement d'un commerce spécifique lié au stationnement des légions frontalières etc. C'est seulement lorsque - et dans la mesure où - l'auteur restitue les règles de son modèle de calcul au contexte historico-géographique du déroulement de l'histoire économique que le raisonnement explicatif peut rendre pertinentes les descriptions et les mesures qui constituent sa base empirique, par exemple les données de statistique archéologique (statistiques des naufrages pris comme indicateurs de l'intensité des échanges, dates et emplacements des trésors enfouis dans les périodes de crise etc. $)^{20}$.

19 Pour une critique sociologique de la «sociologisation sauvage» par les économistes des modèles universels de stratégie, cf. J.-C. Passeron, «Homo sociologicus : réponses à objections », Le Débat (79), mars-avril 1994, Paris, pp. 114-143.

20 K. Hopkins, «Taxes and Trades in the Roman Empire», Journal of Roman Studies (70), 1980, pp. 101-125. 
Mon argumentation vise à montrer que, dans une science sociale, les modèles restent toujours, implicitement ou explicitement, des «modèles à déictiques », c'est-à-dire des modèles qui exigent, pour prendre un sens explicatif, que leur soient associées des descriptions faisant intervenir d'autres informations que celles qui sont simplifiées et sélectionnées pour les besoins de la modélisation. Quand il porte sur des phénomènes sociaux, un modèle, même le plus mathématisé, reste toujours, par le sens historique de ce qu'il permet d'asserter, un modèle à déictiques. On ne peut jamais oublier dans la formulation de son intelligibilité explicative le sens sociologique ou historique, le sens daté et localisé de ce qu'il asserte, c'est-à-dire le sens qu'il tient du contexte singulier où les phénomènes qu'il décrit sont observables. Qu'on se guide (a) sur le «principe de rationalité des actions » en économie, en adoptant la psychologie de convention» qu'elle se donne ou (b) sur la distinction parétienne entre des classes ou genres d'actions caractérisés par leur distance inégale à la rationalité d'action, ou encore (c) sur les différents «types idéaux » d'une « orientation de l'action» construits par le sociologue ou l'anthropologue sur la base d'une comparaison entre cultures, le sens d'un modèle des actions sociales implique toujours qu'on se prononce sur des motifs d'agir, qui ne peuvent eux-mêmes être interprétés que par la comparaison des contextes différents où ces motifs sont plus ou moins opérants. Les déictiques «indexent» toujours (directement ou indirectement) l'argumentation sur des «cas », c'est-à-dire sur des singularités (individuelles ou collectives) solidaires de lieux géographiques et de moments historiques.

Un modèle pur est un modèle universel: ses définitions de «variables » et ses règles de calcul ne peuvent contenir de déictiques, même implicites. C'est un protocole de calcul qui permet de s'assurer, au prix de la délocalisation et de la détemporalisation des hypothèses ${ }^{21}$, qu'on restera cohérent de bout en bout dans la manière dont on compose ou calcule leurs effets. Un modèle universel ne compose que des propositions logiquement universelles, dans une chaîne hypothéticodéductive. Au contraire, dans une science sociale, où la «référence» aux objets ne peut être dissociée de leur singularité, tout commence pour l'explication après qu'on ait «fait tourner le modèle»: l'enquête nomme ce retour à la réalité. Il faut toujours raisonner sociologiquement pour évaluer ce que prouve un modèle, en explorant minutieusement par le retour à des méthodes d'observation le contexte de l'observation et de la mesure. La rencontre entre la «distribution théorique» des valeurs numériques que le modèle donne à prévoir et une «distribution observée » n'ouvre jamais une question à laquelle on puisse répondre par « oui ou non », en termes de «tout ou rien», de «corroboration» provisoire ou de «réfutation» définitive comme dans un test expérimental. J'ai vu, dans une enquête sur la progression du chômage un chercheur considérer que la rencontre entre les séries de chiffres était «encourageante», qu'il y avait un «petit quelque chose» de probant; et tel autre de la même équipe conclure que c'était un «bide», qu'on aurait pu aussi bien utiliser un modèle d'épidémiologie, au lieu d'un modèle de diffusion

21 Il faudrait dire ici, pour être clair, dé-géographisation et deshistoricisation des hypothèses : termes peu prononçables. Il y a bien, dans un modèle pur, référence à l'espace et au temps, mais il s'agit, comme dans le modèle d' Hotelling, de l'espace géométrique (un segment de droite) ou, comme dans un modèle de démographie historique, du temps logique défini par les seules règles et définitions du modèle. 
sociale, pour «simuler» un processus aboutissant aux mêmes résultats chiffrés, bref que la proximité des valeurs «théoriques » et des valeurs «observées » aurait été aussi, ou aussi peu, probante pour établir empiriquement un constat de causalité. Les tests de «significativité statistique» ne tranchent pas, puisqu'ils incluent la clause «à contexte constant ou indifférent» et qu'on peut toujours invoquer l'action perturbatrice de variables dissimulées dans le contexte singulier de la mesure qui sert de test empirique. L'examen de la rencontre entre la logique du calcul et la logique de la détermination par le contexte conduit nécessairement à des raisonnements qui, pour décider de ce que prouve un modèle, doivent sortir de sa stricte logique de calcul.

\section{Les modèles du vote}

«La sociologie électorale est la voie la mieux asphaltée sur le réseau routier de la sociologie quantitative», disait un sociologue de cette discipline. Et en effet rien de plus comparable à travers le temps ou l'espace que la mesure des «oui » et des «non» accordés à un nom ou une liste, et cela que la question soit posée dans un questionnaire de sondage ou par une pile de bulletins dans un bureau de vote. L'histoire et la géographie politiques y trouvent, depuis qu'il y a des démocraties électorales qui fonctionnent comme des questionnaires, une base empirique de mesures comparables en leur simplicité: d'où séries chronologiques et cartographies. À la pauvreté près de l'information - on ne mesure que les choix que mesure aveuglément, à un instant donné, le vote ou le sondage - on reste dans un univers où la commensurabilité semble aller de soi.

Prenons l'exemple des modèles qu'utilisent les sociologues du vote qui veulent calculer des effets probables ou possibles, afin de prévoir des résultats électoraux à partir d'hypothèses quantifiées ou de comprendre après coup l'évolution de valeurs observées. Je pense aux petits protocoles de calcul dont on se sert, pour systématiser l'analyse des résultats électoraux ou pour pronostiquer la formation des votes. Modélisons (comme je m'amusais à le faire un certain samedi 19 avril 1995 avant le premier tour de l'élection présidentielle en France) le pronostic que l'on pouvait formuler, à partir des derniers sondages, sur le vote du lendemain des électeurs qui se disaient encore indécis, ou sur les reports de voix entre les deux tours à venir du même scrutin. Plutôt que de parier au gré d'une statistique subjective ou d'enchaîner, par des raisonnements discontinus, des additions ou des déperditions de pourcentages tels qu'on les a observés dans le passé, on peut construire plusieurs modèles pour calculer en probabilité des résultats possibles en se donnant des hypothèses sociologiques différentes sur le processus de décision des indécis. On reste dans la zone des explications hypothétiques, mais on se donne les moyens d'une totale cohérence logique et statistique dans l'enchaînement des conséquences à partir des hypothèses qu'on a inscrites dans le modèle.

Soit (a) un modèle qui prévoit que la réserve des électeurs «encore incertains de leur vote» se ventilera au prorata des pourcentages déjà dessinés par ceux qui sont déjà certains du leur: pourcentages de départ qu'on peut chiffrer, à une date donnée, pour un scrutin donné, à partir d'un bon échantillonnage de sondage. Le modèle est bâti sur une hypothèse simple: tôt décidés ou longtemps incertains, tous les électeurs de ce scrutin sont supposés avoir la même mentalité politique; au délai d'hésitation près, ces deux sous-ensemble d'électeurs sont supposés être, 
dans leur décision finale, également représentatifs de «l'ensemble parent» des électeurs français. Dans ce modèle, les pourcentages respectifs des candidats, tels qu'ils sont connus pour les électeurs décidés, ne changeront pas, en dépit de l'entrée dans le vote des votants perplexes.

Soit (b) le modèle qui prévoit que ces réservistes, supposés les plus étrangers aux passions partisanes du choix politique, se décideront au final de manière aléatoire entre tous les candidats. Le modèle prête au sous-ensemble des hésitants une indifférence (ou une ignorance) politique portant au même degré sur tous les candidats, par quoi ils diffèrent du sous-ensemble des électeurs déjà décidés. Dans ce modèle, le calcul des conséquences est toujours aussi rapide que certain: les pourcentages finaux changeront au détriment des mieux placés, puisque tous les candidats bénéficieront alors indifféremment d'une part égale de cet abondement. Le classement d'arrivée pourra changer ou non en fonction de l'intervalle qui séparait au départ les scores tels qu'on les a mesurés sur les électeurs décidés: l'effet de ces écarts sur le classement final est alors lui-même calculable.

Soit (c) un troisième modèle qui attribue aux candidats, placés dans un certain ordre par les électeurs déjà certains de leur préférence partisane, des pourcentages différents pour le vote des indécis. On suppose ici que les indécis manifesteront au dernier moment une distribution des préférences différente de l'ensemble constitué par ceux qui se sont décidés les premiers; il faut alors conjecturer les pourcentages propres aux indécis à partir d'une analyse d'antécédents fournis par d'autres enquêtes: on doit injecter plus d'informations dans les données de départ. Le modèle n'a en effet d'utilité que si on peut préciser les préférences des indécis par des probabilités fondées sur une sociologie informée de la dépolitisation ou de l'indifférentisme électoral en France. Dans ce troisième modèle, les pourcentages finaux changeront et, plus probablement que dans le modèle (b), l'ordre d'arrivée.

Chacun des modèles permet de calculer et, on le voit, de pronostiquer un résultat, par des opérations mécaniques qui restent en cohérence avec les principes qu'on a inscrits dans le modèle. Mais le choix entre les trois modèles ne peut reposer que sur des hypothèses d'histoire et de sociologie politiques, sur la connaissance du passé électoral de la population française, sur l'analyse de la conjoncture politique en France etc. Tous ces modèles expriment en les quantifiant des processus possibles dont on a des exemples empiriques. Mais pour pouvoir privilégier un des modèles il faut raisonner sociologiquement sur les exemples, c'est-àdire enrichir la description des «cas » en référant les exemples à des descriptions forcément idéal-typiques des «contextes».

Il se trouve que le premier modèle permettait de prévoir, à partir du premier tour, les reports de voix donnant le score final de De Gaulle et de Mitterrand en 1965. Mais on n'a pas pour autant affaire à un constat susceptible d'être généralisé par induction, puisque la définition de ce «cas» est indissociable d'un «contexte», lequel reste, par définition, impossible à épuiser par une «description définie »: c'est la forme même de la démarche par laquelle on identifie un «cas» historique qui interdit de raisonner sur lui comme sur un phénomène susceptible de répétition, comme s'il n'était qu'un simple «exemple», susceptible d'être remplacée par n'importe quel autre dans une série infinie d'exemples illustrant la même définition d'une relation qui se vérifierait hors de tout contexte.

Est-on plus assuré d'avoir fait l'hypothèse d'une relation plus probable, quel que soit le cas, avec le deuxième modèle, parce qu'il est assez souvent vérifié dans 
des élections triangulaires de second tour, ou plus fréquemment encore dans des élections sans enjeu (par exemple avec le vote nominal pour des candidats inconnus de la plupart des électeurs, à choisir sur une liste pour le renouvellement sans enjeu du conseil d'administration ou d'une Mutuelle)? La distribution aléatoire des votes relève d'une psychologie mécanique de l'indifférentisme (faible information, distribution au hasard des préférences individuelles). Mais il faut alors faire intervenir, dans l'argumentation et l'estimation, des résultats d'enquêtes sociologiques antérieures sur le fonctionnement et la composition d'une institution ou d'une association pour justifier le choix de ce modèle.

Le troisième modèle est le plus exigeant en connaissances et informations préalables. Il suppose pour avoir quelque vertu prévisionnelle que l'on formule et chiffre des hypothèses sur les formes, les degrés et les variables de l'indifférentisme politique dans la conjoncture à laquelle on applique le modèle; il appelle une connaissance historique du passé des mentalités politiques en France, en particulier un suivi de la sociologie électorale de l'abstention sur une assez longue série de mesures opérées dans des cas de figures que l'on contraste ou rapproche par des traits du contexte supposés pertinents pour le vote; une psycho-sociologie particularisée et documentée de l'image des candidats dans le sous-ensemble des hésitants, distincte de celle du sous-ensemble des tôt-décidés, etc. La modélisation est une garantie de cohérence formelle, mais de rien d'autre: elle ne garantit que le moment autonomisé de raisonnement dont elle contrôle l'enchaînement logique; elle ne peut se substituer, dans la prévision comme dans l'analyse, au raisonnement sociologique qui l'englobe et qui guide le choix entre les paramètres du calcul.

Remarquons-le: la situation argumentative ne change pas lorsqu'on possède enfin le résultat final du vote. Il ne donne pas raison à un des trois modèles en termes de «tout ou rien»; il ne «corrobore» pas au sens de Popper la théorie sur laquelle on l'a construit. Le sociologue politique ne peut qu'intégrer ce résultat à sa connaissance historique de la vie politique en France, toujours améliorable par une description plus «dense». La thick description, telle que la décrit Geertz en anthropologie peut toujours être approfondie par une lecture plus poussée de l'intrigue, qui est tout autant déchiffrement qu'observation ${ }^{22}$ Une observation sociologique ne peut que s'ajouter à une série déjà existante d'observations : pour argumenter et prouver, le sociologue ne peut que la composer avec d'autres, en remembrant l'ensemble des connaissances sociologiques qui constituent son contexte et qu'elle rectifie, confirme ou complique. On ne peut pas dire que, forts de ce test empirique, nos trois modèles sont devenus chacun de petites théories susceptibles d'une «réfutation» poppérienne, comme le sont les modèles d'une science expérimentale. Au soir du 21 avril 1995, où le premier arrivé n'était pas celui que désignaient les derniers sondages (Jospin arrivait devant Chirac qui devançait Balladur), on peut sans doute dire que le troisième modèle s'est trouvé être un peu plus prévisionnel (cela ne garantit rien pour l'avenir), qu'il rend mieux compte, en ce cas, de ce qui a bougé dans la mobilisation votante des $12 \%$ de sondés qui ne se sont décidés qu'au tout dernier moment. Ils se sont déterminés, suggère le résultat, quand on le rapporte à ces seuls modèles, selon des proportions

22 C. Geertz, The Interpretation of Cultures (Chap I), New York, Basic Books, 1973. 
qui ont bouleversé l'ordre d'arrivée des trois principaux candidats prévu dans les sondages: en gros les indécis ont préféré Chirac plus que proportionnellement à la préférence des tôt décidés en faveur de Chirac

Pour certains commentateurs, le résultat confortera l'idée qu'au-delà d'un certain seuil d'indifférence politique (de perplexité, de désabusement, de rejet), la psychologie des décisions de l'électeur n'est plus la même que chez les citoyens fidèles à une personnalité ou un parti; que le processus de formation de l'opinion sépare d'une manière statistiquement significative les choix finalement opérés par les hésitants des choix tracés par l'habituation ou l'affiliation politiques. Mais le troisième modèle ainsi «vérifié» n'est pas validé par le résultat du vote comme par une «démonstration». Il ne «prouve» même pas que les électeurs perplexes se sont guidés sur une représentation des candidats très différente de celle des votants plus tôt décidés. Le troisième modèle ne propose une description empirique de ce qui s'est passé meilleure que les deux premières qu'à condition d'introduire de nouvelles hypothèses appelant de nouvelles enquêtes: il faut par exemple supposer que ceux qui se sont très tôt dits certains de leur vote sont euxmêmes restés «scotchés» à leur intention initiale et n'ont pas changé en fonction de la dernière phase du déroulement de la campagne; supposer aussi que leurs premières intentions de vote ne relevaient pas d'une déclaration de façade qui ne les engageait guère, dans l'attente d'une décision réservée à la réflexion, remise à plus tard etc. Bref, la connaissance historique est toujours renvoyée à un approfondissement et à un élargissement de l'enquête sociologique, qui est aussi une reprise et une continuation du récit de la vie politique française. De quelque manière que je veuille raisonner pour expliquer ce qui s'est passé, même à propos d'un comportement aussi univoquement mesurable que celui du vote, je dois recourir à des informations et des formes de traitement de l'observation qui ne figurent pas dans le modèle. Un modèle ne garantit que la forme de raisonnement et la force logique de preuve qu'il soustrait momentanément à d'autres formes de raisonnement et de causalité.

Autrement dit, comme tout modèle, y compris les plus riches en équations liant variables et paramètres, nos trois modèles simplets font voir l'articulation entre deux formes de preuve. (a) Dans un modèle, on ne fait jamais que transcrire la description hypothétique d'un processus sous une forme anhistorique qui permet de calculer des résultats ou, au moins, de déduire des propositions, sous toute garantie de cohérence formelle: l'efficacité aveugle des règles opératoires constitutives d'un modèle assure que la déduction qu'on lui confie sera logiquement impeccable. (b) Mais s'ils contrôlent parfaitement l'exactitude de calculs complexes, que la langue naturelle ne pourrait mener sans risquer d'en perdre le fil, les modèles ne garantissent jamais que des moments et des morceaux du raisonnement sociologique, des conjonctions partielles d'assertions; ils ne peuvent jamais prendre en charge de bout en bout la justification d'une explication causale. Dans une science sociale, le modèle est un instrument d'analyse, non de synthèse; sa confrontation à l'observation oblige le sociologue à continuer son raisonnement par d'autres voies d'enquête pour conceptualiser de nouvelles observations dont la signification contextualisée déborde de toutes parts le langage hypothéticodéductif du modèle.

Aucune science sociale ne peut donc se réduire à une méthodologie des modèles, sauf à se résigner à n'être plus qu'un jeu formel, indifférent à la 
compréhension des phénomènes observés. Une narration ou une exploration ethnographique de terrain, une description sociologique contextualisée ne peuvent évidemment pas «réfuter» un modèle, puisque celui-ci n'asserte que sa propre cohérence hypothético-déductive. Mais aucun modèle ne dispensera non plus une explication de procéder aux enquêtes sociologiques, historiques ou ethnologiques, qui seules fournissent les moyens de formuler au cas par cas, à travers la diversité de leurs observations singulières, la contextualisation de l'explication, que le modèle a dû neutraliser pour pouvoir fonctionner mécaniquement comme protocole de calcul.

Mais ne serait-il pas possible, dira-t-on, d'enrichir un modèle trop pauvre en y intégrant, après formalisation, de plus en plus de descriptions fournies par les sciences de l'exploration empirique? Construire un modèle nourri de nouvelles variables absorbant de proche en proche l'univers opaque des déterminations «exogènes » constitue, on le sait, le programme du savant modélisateur lorsqu'il est insatisfait du rendement de ses variables «endogènes». Quelles variables oubliées faudrait-il introduire dans les modèles explicatifs de la «fécondité» demandent souvent les démographes aux sociologues quand ces modèles n'ont pas prévu ou ne rendent pas compte de chutes ou de pics de natalité dans l'histoire des populations? Las! l'exploration empirique du cours du monde historique étant interminable et discontinue, voilà le modélisateur en train de courir, d'ajouts en ajouts toujours insuffisants, après une chimère de l'argumentation: rien moins que l'absorption du contexte par le modèle, c'est-à-dire l'impossible absorption des langages naturels de la comparaison historique dans le langage formalisé d'un modèle universel ou d'une équation finale.

\section{Déictiques et variables}

Revenons à nos «déictiques », par quoi j'entends tous les signes du discours qui dans un énoncé réfèrent le sens de l'information qu'ils donnent à la situation d'énonciation, agissant ainsi sémantiquement par «désignation» et non par «description définie »- lorsqu'ils associent, fût-ce en partie, une désignation datée et localisée à un énoncé de propriétés dans une définition universelle. Un déictique, rappelons-le, est un signe qui doit, pour signifier quelque chose, être référé à des termes qui le précèdent ou le suivent dans le discours et, plus généralement, à toutes les «circonstances» qui l' «ancrent pragmatiquement» dans une situation de parole déterminée. Si je suis à une station de bus où il y aussi des barrières métalliques pour contenir le public et que j'entends la foule s'écrier: «Il arrive !», il faut que je regarde autour de moi l'ensemble de ces circonstances pour savoir si ce « il » désigne le bus ou le Président de la République. Dans l'explication que fournit une science sociale, un modèle à déictiques est un schéma mental dont la fonction est d'expliquer ou de maîtriser, en la simplifiant pour la raccourcir, la description d'une configuration singulière. Empruntons l'exemple d'un modèle à déictiques aux schémas de représentation de l'environnement géographique dont nous usons tous pour nous orienter dans les déambulations de la vie quotidienne.

J'ai ainsi utilisé pendant longtemps, lorsque je parcourais à pied les montagnes du Midi de la France, d'un modèle visuel qui me permettait de prévoir les formes et la viabilité des pentes. C'était celui d'un schéma de paysage où, comme pour la montagne Sainte Victoire, le versant nord boisé et en pente douce contraste avec 
la face sud, abrupte, rocheuse et dénuée de végétation. Ce modèle de montagne, où un $u b a c$ à la fois ombreux, humide et adouci en ses profils tranche par tous ses traits avec un adret ensoleillé à la végétation rare et desséchée, riche en aplombs et falaises, m'a longtemps servi à m'orienter dans le choix de mes trajets comme dans l'anticipation de l'aspect des paysages que je n'apercevais pas encore. À chaque confirmation nouvelle sa cohérence formelle me rendait plus «compréhensible» l'association de tous les traits géologiques et zoologiques qu'il répartissait en deux séries symétriques de corrélations. Mon modèle de Sainte Victoire a longtemps «marché »; il s'exemplifiait à plus de $90 \%$ des cas dans l'échantillon de mes déambulations alpines du Rhône aux Alpes-Maritimes: $\chi^{2}$ merveilleusement significatif ! Le sens des relations qu'il corroborait me semblait parfaitement intelligible. La distraction encourage l'illusion d'universalité, chez le sens commun comme dans la pensée scientifique.

J'étais au bord de penser, tout simplement parce que je n'y avais jamais pensé explicitement comme à un modèle, que tous les paysages montagneux du monde étaient prêts à obéir à ce modèle où l'ensoleillement semblait traîner après lui toute la brutalité du paysage peint par Cézanne qui s'y était associé dans mes observations réitérées. Ce n'est qu'assez tard, et parce que je pensai à ma représentation comme à un modèle, que la théorie qu'il impliquait m'obligea à me mettre en quête de contre-exemples. Je les trouvai sans difficulté en me promenant dans d'autres contrées: simple usage pédestre de la méthode comparative. Le soleil, découvris-je, ne s'associait à la verticalité des parois que dans des lieux particuliers du globe terrestre, là où le sens d'une poussée géologique datée et localisée avait basculé le plissement du nord vers le sud. Ailleurs dans le monde, ces deux variables n'étaient pas en colinéarité de variation. L'orientation de l'ensoleillement dépendait d'un «déictique». Qu'il faille ajouter au modèle universel un déictique limitatif ne m'était devenu concevable qu'à partir du moment où j'avais pu analyser autrement, par la comparaison de cas, une configuration singulière qui m'était trop familière. Référé à des précisions de date et de lieu, le modèle n'était plus universel, mais il était devenu plus exact en imposant aux énoncés la considération pertinente de quelques noms propres désignant une aire géographique. Victime du langage de l'universalité nomologique, j'avais longtemps omis d'apercevoir l'historicité singulière du plissement alpin, indispensable pour parler un peu plus véridiquement des formes de la Sainte Victoire.

\section{LES USAGES DU «PRINCIPE DE RATIONALITÉ» DANS L'ANALYSE DES STRATÉGIES}

Le rôle problématique du «principe de rationalité » dans l'explication des stratégies avait été exploré par Vilfredo Pareto qui, dans son Traité de sociologie générale, fondait sa sociologie sur une distinction radicale entre les «actions logiques» et les «actions non-logiques » (Ière et IIème classes) ${ }^{23}$. C'est dans les

23 V. Pareto, Traité de sociologie générale (1ère éd. italienne, en 2 volumes, 1916; 1ère éd. française, revue par l'auteur, en 2 volumes, 1917-1919); 3ème éd. française (G. Busino ed.), Genève, Droz, 1968. 
subdivisions de la deuxième classe d'actions que commence la sociologie, avec l'interrogation sur les actions «pseudo-logiques» des acteurs sociaux, qui trouvent toujours des raisons «logiques» à invoquer pour justifier leurs décisions. Pareto disait «vernis logique» pour caractériser les raisonnements fallacieux mis en œuvre dans l'argumentation propre aux «dérivations» qui expriment sous ce «voile» les «résidus » non-logiques de l'action sociale. Disons plus simplement ratiocination, pour nommer en ses diverses formes cet usage biaisé de la rationalité: usage dysfonctionnel pour la rationalité du raisonnement scientifique, mais fonctionnel pour les passions du raisonneur, C'est cette ambivalence inscrite au cœur de l'usage de la Raison qui fait la difficulté rencontrée par tous les modèles de la «décision rationnelle». Au lieu d'abandonner l'ambivalence au non-sens Freud y voyait le principe agissant des «rationalisations» du Moi, de même que Marx voyait dans la dissimulation ou la déformation du monde réel le principe actif des «idéologies».

\section{Décision politique et décision calculée}

C'est dans la sociologie politique que l'on voit le mieux l'ambiguïté des modèles de décision qui supposent que les actions peuvent toujours être expliquées comme des «stratégies » ${ }^{24}$. L'exemple privilégié que donnait Pareto de l'action logico-expérimentale n'était pas, on le sait, celui des agents économiques, producteurs ou consommateurs, mais celui de l'ingénieur qui calcule le projet de construction d'un pont en fonction des connaissances qu'il possède sur la résistance des matériaux. «Les actions étudiées par l'économie politique » ne sont jamais citées chez lui qu'en second rang, comme si leur teneur en connaissances logico-expérimentales était déjà moindre. Comme le dit l'auteur, elles ne font qu'appartenir «en très grande partie à cette classe». L'exemple que prend le plus souvent Pareto d'une action économique qui fonctionne pleinement comme une action logique est d'ailleurs révélateur, c'est celui du spéculateur, non celui de l'agent économique tout-venant, producteur ou consommateur sur un marché. Le spéculateur, en effet, a toute la pureté d'un «cas d'école». Il n'a qu'un seul but: gagner le plus possible d'argent. Il vise un but toujours commensurable en termes monétaires aux coûts et aux investissements qu'il consent; il n'en change jamais dans ses enchaînements de décisions. Toute son activité de rassemblement d'informations est subordonnée à ce but unique.

Parmi les actions logiques, Pareto ne faisait intervenir qu'en troisième rang c'est-à-dire à un titre encore moins pur que les décisions du spéculateur, «un certain nombre d'opérations militaires, politiques, juridiques $»^{25}$. Les actions $\mathrm{du}$ chef de guerre calculant une manœuvre sur le champ de bataille, celles de l'homme d'Etat appliquant les principes de la Realpolitik ou celles du chef politique anticipant en sociologue informé les effets sociaux que produiront en toute probabilité ses décisions ou règlements compte tenu des attitudes des justiciables (y compris en leurs réactions non-logiques), relèvent bien, selon Pareto, de la

24 J.-C. Passeron, «Pareto: L'économie dans la sociologie» in Economia, sociologia e politica nell'opera di Vilfredo Pareto (sous la dir. de Malandrino C. e Marchionatti, R.), Firenze: Leo S. Olschki Ed., 2000, pp. 25-71.

25 Cf. dans le Traité, op. cit. le tableau classificatoire de départ, p. 67. 
«stratégie», c'est-à-dire du raisonnement logico-expérimental. Mais c'est seulement à deux conditions, fortement restrictives: premièrement que le calculateur soit un «machiavélien » accompli, indifférent à toute autre considération que celle de l'efficacité de ses décisions; et deuxièmement, qu'une fois son objectif ou sa hiérarchie des objectifs fixée, il puisse sélectionner ses moyens d'action en rapportant leur efficacité à leur coût, ce qui suppose évidemment la possibilité de commensurer (ou au moins celle d'ordonner) les valeurs ainsi comparées. Ce que nous appellerons l'axiome de commensurabilité des valeurs entrant dans un calcul est indissociable de la définition d'une action logique et, donc, de tout plan mettant en œuvre une stratégie.

Le choix que faisait Pareto de prendre pour objet l'hétérogénéité ${ }^{26}$ des données sociales accessibles à l'observation historique est, à mon avis, constitutif de toute sociologie. Ce constat suffit à exclure du langage de l'explication sociologique la facilité verbale qui s'est répandue dans toutes les sciences sociales et qui consiste à baptiser et à traiter comme «stratégie» n'importe quelle action sociale.

Roland Barthes avait donné le signal de cette mode sémiologique dans ses Mythologies où il majorait allégrement le sens explicatif des «sémantisations de l'usage »- déjà cataloguées par les linguistes - en donnant à comprendre comme des stratégies visant à constituer ou renforcer une identité sociale les représentations les plus courantes de la vie quotidienne des années' 50': le beefsteak-frites à la française, la photographie de presse dans les magazines à grand tirage, le combat de catch vu à la télé, etc. Il identifiait ainsi sans autre forme de procès le sens social d'un acte et la stratégie symbolique de l'acteur; autrement dit, il confondait, comme l'ont fait alors remarquer Buyssens puis Prieto, sémiologie de la signification et sémiologie de la communication. Ou, comme le formulerait le sociologue, les raisons de faire et les raisons de dire. Se représenter ou dire que «Ça fait (bourgeois, beauf, intello, peuple, etc)» de rouler dans un type de voiture plutôt qu'un autre suffirait-il à épuiser les raisons pour lesquelles on choisissait dans les années ' 60 ' de s'acheter une GTI, un 4x4, une deux-chevaux ou une dauphine?

Mais, depuis lors, que n'a-t-on pas analysé comme «stratégie»? Des bataillons de sociologues ont épluché jusqu'à la trame les biographies d'hommes et femmes, illustres ou repêchés en archives, afin de reconstruire leurs péripéties et leurs rencontres en stratégies de la mobilité sociale, de la réussite intellectuelle ou littéraire, sans oublier les stratégies du poète maudit ou de l'écrivain raté. De même, avance-t-on beaucoup dans l'explication causale de l'usage que font les «assistés» des systèmes contemporains de protection sociale en l'interprétant comme un calcul d'utilité que feraient rationnellement les «exclus», qui se refuseraient à sortir d'un chômage indemnisé trop près des plus bas salaires, parce qu'ils rapporteraient le «gain marginal» d'un vrai salaire au «coût» qu'ils devraient consentir en travaillant à plein temps plutôt que pas du tout? Ou en interprétant comme une «optimisation » bien calculée l'enfermement des assistés dans une dépendance rationnellement choisie? Que fait-on, dans cette explication causale, de la diminution de la «valeur» culturelle accordée au travail, dévalua-

26 C'est ce terme parétien qui définit le mieux la différence des tâches entre le Cours d'économie politique et le Traité de sociologie générale. Cf. sur Pareto sociologue de la complexité, G. Busino, «Pareto redivivo» in Economia, sociologia e politica... op. cit., pp. 1-24. 
tion que l'enquête sociologique permet de voir se répandre en fonction des nouvelles conditions de la socialisation (familiale et scolaire) des jeunes générations? Peut-on se contenter de décrire la démission des familles les plus démunies face à l'Ecole, ou l'insoumission des enfants les plus étrangers à la culture scolaire, comme des «stratégies sociales» de joueurs informés qui optimiseraient les formes de leur révolte ou de leur résignation par un calcul de leurs gains ou pertes probables en fonction de leurs informations sociologiques sur leurs chances de réussite à l'école?

J'ai même rencontré des théoriciens des jeux capables d'expliquer les choix les plus passionnels par un calcul rationnel: par exemple des emportements de rage religieuse comme la quête du martyre volontaire chez les chrétiens montanistes de la fin du $\mathrm{II}^{\mathrm{e}}$ siècle. Si l'on explique le comportement montaniste par un modèle du choix rationnel, on affirme que les montanistes, virtuoses de la maximisation individuelle du profit spirituel, auraient calculé, bien avant le calcul que donnera Pascal de ce pari, que le martyre, qui garantit la certitude du Paradis aux témoins de la vraie foi, procure immédiatement un gain infini sans commune mesure avec les gains finis engrangés en ce monde, et cela au coût minimal en dépense de temps, en vertu patiente, en bonnes œuvres etc. Lorsqu'il m'arrive de me poser des questions sur les causes de l'apparition du montanisme, je préfère encore relire Renan, ou d'autres historiens des origines du christianisme: j'avoue que je «comprends » mieux alors le sens de cette piété suicidaire dans des travaux qui me décrivent son «contexte», son émergence liée à des particularités culturelles et socio-économiques de son lieu géographique de première diffusion (la Cappadoce, évangélisée par Paul), la déception d'une attente toujours allongée du Royaume promis, l'extension rapide de ce courant extrémiste, face à la persécution impériale d'une secte adventiste d'immigrés, jusqu'en Afrique du nord et en Gaule, et enfin la réaction politique de l'Eglise romaine qui finit par se débarrasser de ces illuminés en condamnant leur piété à l'hérésie - car comme le remarque humoristiquement Weber, une hiérarchie ecclésiastique a, elle, un intérêt rationnel, différent de l'intérêt religieux des croyants à "garder au moins quelques fidèles» dans l'institution. Pourquoi sociologues, anthropologues et historiens iraient-ils emprunter aux économistes la «psychologie de convention» dont ceuxci ont besoin pour faire tourner - au minimum de sens psychologique et donc de vertu explicative - leurs modèles de l'action? Dans les sciences de l'intelligibilité historique, la reconstruction du sens des actions vise toujours à une forme d'explication particularisée: il leur faut choisir leurs hypothèses, non pas en fonction des possibilités de calcul abstrait qu'elles ouvrent, mais en fonction de la compréhension singulière des actes qu'elles procurent en les rattachant à leur contexte historique et anthropologique.

\section{Jeux et règles du jeu}

Le maniement politique du concept de stratégie incite à la prudence méthodologique. Mais où commence l'aspect politique d'une décision? Petite ou grande, une décision a toujours quelque chose du choix politique. Une décision est «politique » en ce qu'elle n'a pas, comme le Dieu de Leibniz, l'éternité devant elle pour répertorier et combiner. Elle n'a pas le temps de calculer et de pondérer ses « coûts d'information » afin d'optimaliser la « désutilité » qu'elle consent pour rechercher 
une information plus complète. Il en va de même pour les «coûts de transaction» etc. Bref une décision politique doit tenir compte de données de l'environnement et d'états des interactions en cours, non énumérables dans une description exhaustive, par là jamais susceptibles d'entrer dans une combinatoire stricte. La théorie «pure» d'un jeu peut, elle, immobiliser, au moins pendant le temps nécessaire au calcul, la mesure du rapport entre le gain et la perte, entre les enjeux et les coûts consentis. Pour seul exemple du flottement de la description que révèlent dans le déroulement d'un affrontement singulier les descripteurs théoriques d'un jeu lorsqu'on veut les formaliser dans un modèle, pensons à la «théorie des dominos » qui fut utilisée un temps par les géopoliticiens de l'empire américain pour prévenir la chute en chaîne de ses protectorats militaires dans le monde.

Entre être agi et calculer, entre décision sauvage et décision rationnelle, Pareto a vu le lien paradoxal: la guerre est un conflit où l'enjeu de redéfinition noncoopérative des règles oblige le joueur rationnel à improviser à chaque coup la décision capable de transformer, par le changement des règles du jeu, un maximum des forces de l'adversaire en faiblesses, et l'inverse dans sa propre donne. Ici, les anticipations rationnelles ne cessent de fluctuer puisque chacun doit rationnellement anticiper chez l'adversaire les effets d'une recherche de l'infraction aux règles du jeu, identique à la sienne propre en sa recherche du coup efficace. La violence guerrière est un jeu où le joueur rationnel doit prendre en compte jusqu'à l'irrationalité du renoncement à tout calcul rationnel, toujours possible chez l'adversaire. La rationalité des choix guerriers s'affronte, comme à sa limite, à la menace d'un coup entièrement irrationnel, qui pourrait devenir efficace si on n'en a pas prévu la parade et fait savoir qu'on l'a prévue. Cette «dissuasion» ne fournit pourtant qu'une stabilisation incertaine et précaire des limites de l'espace du jeu guerrier. Comment calculer, même en probabilité, les limites admises en commun d'un affrontement, si on ne dispose pas d'une information sur les limites de la «folie» possible de l'adversaire, ou, si l'on préfère, sur les règles particulières de sa rationalité d'action? Le scénario d'une guerre n'est pas, comme un scénario de western, réglé par la tradition d'un genre où l'on peut anticiper ce qui va se passer si le joueur perdant dégaine son revolver ou renverse d'un coup de pied la table de poker.

Une véritable partie politique ou militaire se joue dans un espace de raisonnement où tous les éléments que la théorie des jeux a besoin d'immobiliser pour se formuler et se formaliser - moyens et fins, coûts et enjeux, pertes et gains, ressources et solvabilité des joueurs, communication et dissimulation (par défausses, double langage, ruse), «bruit» et «information»- changent continûment de «valeur» dans la supputation et les anticipations des joueurs. Appliquée à toute interaction sociale, à tout rapport de force non strictement réglementé, la notion de «jeu» n'est qu'une métaphore de la perfection du calcul. S'il est utile de faire fonctionner cette métaphore comme un modèle, c'est précisément parce que cette rationalisation par passage à la limite permet de faire apparaître, lorsque le récit historique révèle l'inadéquation descriptive du modèle, des propriétés fondamentales de l'interaction politique, et plus généralement de toutes les interactions qui, en admettant une redéfinition perpétuelle des règles de la partie, favorisent par là l'escalade de la violence. L'histoire des conflits et des alliances, des automatismes 
du quotidien et des trouvailles fécondes n'est pas un jeu au sens strict, ni une agrégation de jeux ${ }^{27}$.

Est-il certain que la plus grande rationalité, définie comme efficacité maximale dans la recherche d'un optimum, appartienne toujours au cynique capable de calculer ses actes par rapport au seul souci du gain de la partie, sans plus entretenir d'autre rapport que purement instrumental avec les croyances et les normes sociales, celles des autres joueurs, partenaires ou adversaires, comme les siennes propres? Suffit-il au machiavélien achevé de savoir mettre à distance ses propres affects comme ceux d'autrui, pour en calculer les effets en toute lucidité? Lui suffit-il de savoir manipuler techniquement tous les acteurs et actes avec la froideur du joueur professionnel? Peut-il se contenter d'obéir rationnellement, avec une constance parfaite dans toutes ses anticipations, aux seuls impératifs du calcul stratégique, pour l'emporter à tous coups sur tous les autres types d'acteurs?

Dans l'histoire, la réponse est moins simple que dans un modèle de théorie des jeux. Lorsqu'il compare les effets des différentes stratégies d'influence, l'historien est obligé de constater que le chef charismatique dont la conviction irréfléchie est d'une seule pièce a souvent été plus efficace (sur les foules, les opinions et les mouvements de masse) que le chef calculateur. Le prophète obsédé par sa mission, le tribun populiste qui colle aux passions de son auditoire ou le despote obnubilé par l'exercice du pouvoir absolu, ces acteurs maniaques sont sans doute aveugles à la logique comme à l'esprit expérimental par leur sensibilité aux pulsions irréfléchies. Mais ils ont souvent imprimé sur les mentalités et les événements une marque plus durable que l'homme d'Etat réaliste et informé. En observant les stratégies à l'œuvre dans les affrontements guerriers ou les négociations diplomatiques, les historiens comme les stratèges ont été conduits à se demander si l'irrationalité, la conviction passionnée ou l'entêtement étaient nécessairement moins payants que les raisonnements conduits rationnellement dans «les eaux froides » du calcul politique décrit par Machiavel.

Loin des tables de bridge, les hommes politiques passionnés ou butés déconcertent assez les prévisions de leurs adversaires pour gagner des parties par d'autres chemins que ceux du calcul, en tout cas, pour marquer des points contre des calculateurs du type des joueurs d'échec ou de bridge. Khomeyni, Sadam ou Milosevic sont-ils des joueurs moins «rationnels», c'est-à-dire moins efficaces par rapport à la réalisation de leurs objectifs, qu'un staff de spécialistes en rationalité technique additionnant leurs moyens d'information et de calcul au service d'une Présidence américaine? Maximise-t-on toujours l'information pertinente en s'aidant des meilleurs experts (militaires ou géopolitiques), en s'armant des meilleures technologies de production ou de destruction, en s'appuyant sur les meilleurs logiciels d' «intelligence artificielle» lorsqu'on fait face à des adversaires qui fondent autrement leurs anticipations et leurs décisions dans la conduite d'un conflit: paris intuitifs, expertises sans règles formulables ou constantes, familiarités d'accoutumance avec le terrain, rapport fluctuant à l'enjeu, immobilisme patient dans la négociation, opiniâtreté dans le refus du compromis, ou

27 Les économistes dits «institutionnalistes» ont re-découvert, au cœur même de l'histoire économique, «l'indépendance» de certains facteurs de la réussite des innovations techniques par rapport au calcul anticipé de leur rentabilité, en la formulant comme path dependency: «dépendance » expliquant ex post le succès d'une innovation par la place qu'elle occupe dans une succession. 
même coups irrationnels du desperado qui n'a plus rien à perdre ${ }^{28}$ ? À quel terme le bilan d'efficacité (et de rationalité) de ces différentes manières de s'affronter doit-il être tiré pour trancher de leur rationalité?

On ne prêche efficacement que des auditoires déjà convaincus, ont répété à l'envie les sociologues de la prophétie ${ }^{29}$. Ce ressort non-logique de l'efficacité symbolique s'observe parfaitement dans la dépendance du leader charismatique par rapport aux données de la situation locale où se fonde son pouvoir. Dans quelque domaine d'influence que ce soit (religieux, politique, guerrier, intellectuel) les leaders dont tout le calcul politique se réduit au principe d'entêtement ou de ressassement, les meneurs dont la stratégie découle d'une proximité affective avec un auditoire particulier et d'une longue familiarité avec l'information locale, anticipent plus exactement les réactions de leurs troupes, ils prêchent plus efficacement leurs opinions publiques, entraînent ou galvanisent mieux leurs exécutants ou leurs masses que les chefs trop subtilement machiavéliens ou les experts universitaires en géopolitique, enchaînés à la valeur universelle de leurs principes stratégiques peaufinés en cabinet. Constatons à tout le moins que les prophètes millénaristes ou inspirés, les chefs monomaniaques ou imprévisibles ont une stratégie plus offensive - qui s'est avérée parfois plus suicidaire mais parfois aussi plus payante - que les calculateurs machiavéliques, trop occupés au trop long calcul de la décision optimale. De tels monstres froids ne sont pas assez ouverts à l'improvisation pour oublier d'être machiavéliques et revenir, en fonction des circonstances, à un usage spontané de leurs affects. Sur un terrain de sport ou de bataille comme à une table de jeu, il arrive que la naïveté déconcertante, l'improvisation, le coup d'audace se révèlent plus «utiles » à l'obtention d'un gain que la rouerie ou la combinatoire patiente.

Le machiavélien qui vise à la perfection machiavélienne - à l'efficacité maximale - se trouve placé ici devant un défi de la rationalité politique. Pour s'accomplir en hyper-machiavélien, il ne doit pas hésiter à user machiavéliquement de sa propre personnalité: il devrait être capable de prendre la décision d'oublier le calcul sur une simple intuition non calculé et, en somme, d'être assez manipulateur pour se laisser manipuler par ses propres affects ou croyances. Et cela, on ne peut jamais mieux le faire que quand on est capable d'oublier qu'on est en train de le faire. Performance machiavélienne difficile à atteindre par la seule vertu du machiavélisme, et pourtant accessible, dès l'enfance, à l'illogisme des mouvements spontanés, fréquent jusque dans les petites altercations de la vie quotidienne. L'oubli, politiquement «utile», des raisons cyniques qu'on a de faire un choix politique pour le ressentir comme un devoir obscur de vengeance ou un

28 Une théorie de l'action politique doit en effet considérer comme une décision toute action qui implique l'engagement de «ressources » de la part d'acteurs par référence à un objectif. Même non-pensés stratégiquement, l'immobilisme ou l'escalade restent des décisions: cf. P. Bachrach et M.S. Baratz, «Two Faces of Power », American Political Science Review, 56, 1962, pp. 947-952; et «Decisions and Non-Decisions: An Analytical Framework», loc. cit., 57, 1963, pp. 632-642.

On trouve la formulation la plus brève de ce paradoxe logique, qui est en même temps une banalité sociologique, chez Schumpeter: "Il suffit d'imaginer ce qui se serait produit si la Jihad avait été prêchée aux 'pêcheurs de Galilée' et aux 'petites gens' de Palestine. (...) Inversement, si Mahomet avait prêché l'humilité et la soumission à ses cavaliers bédouins, l'auraient-ils écouté ou lui auraient-ils tourné le dos?» (J. Schumpeter, Impérialisme et classes sociales (trad.), Paris, Minuit, 1972, p. 81-82). 
mouvement irrépressible de pitié n'est pas psychologiquement impossible, loin de là. Il suffit de consulter les figures par lesquelles la théorie psychanalytique décrit sur une base clinique la structure interne des actions psychiques: ambivalence, sublimation, dénégation, déplacement, contre-investissement, etc. Il n'y a pas que les romans noirs américains où l'on puisse voir se manifester l'efficacité psychopathologique de détectives, indifférents aux moyens de police scientifique comme aux déductions abstraites de roman policier, seuls capables pourtant de prévoir le comportement de serial killers psychopathes, parce qu'ils peuvent s'identifier à leur proie à partir de leurs propres refoulements et fantasmes.

\section{Cynisme, naïveté, conviction menteuse}

L'usage de la ruse florentine - celle dont Machiavel entendait faire une science - relève d'une rationalité qui peut entrer sans difficultés dans un modèle stratégique, tant qu'il ne s'agit que de rétention d'information, de mensonge, de simulation ou, plus généralement de toutes les décisions consciemment réfléchies qui s'avèrent efficaces pour faire faire chose au partenaire ou à l'adversaire. Mais on ne peut s'arrêter là dans la recherche d'un maximum d'efficacité politique, fondé sur une utilisation rationnelle de tous les moyens accessibles. Il ne manque pas de situations - dans la propagande politique et la prédication religieuse par exemple - où celui qui joue la conviction par calcul - avec une «idée de derrière la tête» comme disait Pascal de «l'habile» en politique - ne peut jamais imiter à la perfection la «présence» de celui qui est convaincu de sa cause au premier degré. Le cynique, l'homme $d u$ second degré qui entend subordonner toutes ses décisions au calcul des moyens efficaces, ne congédierait-il pas une part de son efficacité en congédiant toute conduite au premier degré?

En bonne stratégie, le calculateur doit, lorsqu'il peut en tirer les ficelles, utiliser cyniquement l'ardeur sans état d'âme des naïfs, néophytes ou compagnons de route. L'efficacité politique d'un raisonnement au second degré est manifeste dans l'anticipation rationnelle: le stratège machiavélien est rationnel quand il utilise logico-expérimentalement les connaissances dont il dispose sur les actions nonlogiques des autres acteurs, adversaires ou alliés, qui n'agissent qu'au premier degré. Mais est-ce suffisant pour atteindre au summum de la manipulation machiavélienne? Evidemment non, puisque la manipulation retorse d'autrui n'est pas le dernier mot de l'efficacité politique, celle-ci n'étant pleinement définie que par la nécessité logique d'agir sur tout moyen qui permet une action utile à la réalisation de l'objectif. S'il découvre qu'il pourrait encore gravir quelques degrés dans la manipulation des actes accomplis au premier degré, l'hyper-machiavélien ne peut rester inerte. Dès qu'il envisage toutes les efficacités logiquement possibles, l'acteur rationnel que suppose la théorie rencontre donc le devoir logique d'appliquer un raisonnement de second degré à sa propre psychologie, s'il peut y découvrir une compétence ou une croyance capables de fonctionner au premier degré au service de sa stratégie,

Dira-t-on que la manipulation intéressée de ses propres convictions constitue un troisième degré de l'anticipation rationnelle? Mais celui-ci ne fonde une stratégie politiquement efficace que pour autant qu'il redevient psychologiquement indiscernable du premier. Et si l'on peut décrire la conviction non calculée comme un principe d'action fonctionnant au premier degré, celui-ci ne donne pourtant les 
moyens d'une stratégie efficace que lorsqu'il parvient à brouiller la représentation des degrés supérieurs du calcul dans les anticipations de l'adversaire. L'art d'être irresponsable ou imprévisible, par lequel l'homme politique qu'on dit «capable de tout » redevient le croyant naïf qu'il est capable de redevenir à l'occasion, peutil entrer dans un «modèle » qui doit ordonner sur une échelle les degrés de l'anticipation rationnelle comme il ordonne les préférences? Les chefs dotés d'une conviction sans failles sont redoutablement efficaces, du moins dans les interactions sociales où la conviction du premier degré ne s'imite que difficilement: actions sur les passions et les actions du plus grand nombre.

Diderot avait posé la question de l'efficacité théâtrale de la naïveté et de la composition, aussi décisive en politique qu'au théâtre, dans Le paradoxe $d u$ comédien. Pascal plaçait le summum de «l'habileté » politique dans la capacité à avoir une «idée de derrière la tête»: celle par exemple que «la guerre civile est le plus grand des maux». C'est en effet cette idée qui permet à «l'habile» de considérer que, tous comptes faits, les «opinions du peuple sont saines», puisque son habileté réaliste ou cynique lui permet d'anticiper en sociologue chrétien l'effet socialement destabilisateur des raisonnements tenus par le «demi-habile». Celuici en effet, sceptique ou libertin, risque toujours d'ébranler la croyance naïve du peuple à l'égard du caractère «naturel» des «grandeurs d'établissement» en lui révélant imprudemment «l'arbitraire de la coutume» qui fonde l'ordre établi et préserve des catastrophes révolutionnaires. Mais cet habile-là serait-il un propagandiste efficace de la défense de l'ordre social en cachant au peuple le raisonnement au deuxième degré par lequel il lui donne raison? La compétition entre meneurs semble souvent, dans la concurrence pour la popularité, favoriser le succès d'un autre cynisme, celui des cyniques naïfs, de ces cyniques malgré eux, que les Grecs appelaient «démagogues », et que l'on dit aujourd'hui «populistes». C'est donc bien la rationalité politique qui commande au machiavélien de traiter sa propre psychologie comme un simple moyen de sa propre stratégie: le coup de maître du manipulateur est de ressentir vraiment les sentiments qu'il veut voir le partenaire, adversaire ou destinataire, lui prêter, et non de les jouer.

La conclusion est inévitable: si la bête la plus efficace est celle qui ne joue pas la comédie de la bêtise comme un rôle de composition, la stratégie d'influence la plus efficace est de réussir à oublier qu'on fait la bête au moment où on s'abêtit le plus. De réussir à oublier qu'on régresse vers irrationnel en se persuadant qu'on progresse dans un raisonnement: moyen le plus efficace d'en persuader autrui. À la source des violences collectives du passé le plus lointain comme au principe des grandes catastrophes contemporaines, on retrouve toujours comme accélérateurs ou amplificateurs des ruptures historiques de la continuité ou de la coexistence sociales, de tels représentants de la sauvagerie semi-calculatrice. Les bêtes politiques associent-elles dans un calcul l'extrême du calcul à l'extrême de la violence irraisonnée? Ou ne sont-elles que des hybrides typologiques oscillant entre influence charismatique et zweckrationalität? Saints et prophètes, conquérants et massacreurs alternent-ils selon le moment des humeurs psychologiques contradictoires ou sont-ils de perpétuels ambivalents? Et s'ils alternent, est-ce au hasard des stimulations qu'ils subissent ou en fonction de leur analyse de la partie en cours? Celui qui alterne ses principes de décision en laissant transparaître son professionnalisme d'acteur devient vite inefficace face à un adversaire, même sans autre force que de l'avoir percé à jour. Pie VII prisonnier de Napoléon à Vincennes 
pouvait encore désarmer la gesticulation théâtrale de l'Empereur d'un simple soupir: Comediante!... Tragediante! La régression d'un führer qui parvient à entrâ̂ner une foule dans son délire, le trucage de ses miracles par le thaumaturge qui y croit en même temps autant que ses miraculés, l'abandon spontané du démagogue à la sincérité menteuse sont autrement efficaces que la gesticulation monotone du prêcheur de métier. Face à un Le Pen par exemple, et devant des téléspectateurs qui enregistrent au premier degré les points marqués sur le ring du petit écran, journalistes habiles et politiques tacticiens, bardés de chiffres et de solides arguments, ont goûté à leur détriment, dans les sondages d'après l'émission, les fruits amers de la tranquille supériorité rhétorique de la conviction menteuse sur le raisonnement honnête et bien informé. Le calcul politique du machiavélien doit logiquement le conduire à se contredire quand il ressent simultanément le besoin de donner libre cours à ses pulsions et la certitude non-raisonnée que cet abandon des règles du calcul lui est profitable. Il se contredit en tant que calculateur, mais l'obligation de non-contradiction et de constance logique dans les principes de l'action est un impératif de morale kantienne, non de stratégie politique.

Concluons: l'opacité de chacun à soi-même et de soi à autrui fonde une efficacité qu'on peut dire à la fois d'un degré inférieur et supérieur à l'efficacité qui coordonne des anticipations bien informées. Or un système formel ne peut pas plus introduire dans la définition d'une stratégie des degrés d'anticipation qui se renversent au gré de l'analyse qu'une hiérarchie des préférences qui se désordonnent dans le cours d'une transaction. La violence, la menace de violence, la dissuasion, sont souvent d'autant plus efficaces que les actions qui y recourent sont plus imprévisibles, plus équivoques et donc moins accessibles à la formalisation. L'action politique est le terrain sur lequel éclate le mieux le paradoxe inhérent à toute application du principe de rationalité à l'explication ou à la prévision des actions sociales: ici, la rationalité informée, fondement théorique de l'action efficace peut encore s'accomplir dans les opérations où elle échappe, d'une manière imprévisible à l'adversaire, à la rationalité du calcul.

Mise à la question de ce qui fait l'efficacité des stratégies politiques, la rationalité voit se multiplier et se désagréger les figures canoniques de son calcul. Toutes les difficultés renvoient à la question de l'articulation entre les aspects stratégiques et les aspects non-stratégiques du comportement politique. Si les affrontements du type «poker» sont, par la structure psychologique des décisions qu'on y enchaîne, les plus proches des interactions conflictuelles de la politique ou de la guerre, c'est qu'ils sont, comme elles, plus rebelles à la formalisation que les jeux de stratégie tels que le bridge ou les échecs dont les règles de gain ou de perte sont formulables sans aucune référence aux rapports non-réglés entre joueurs.

Les retournements inattendus du psychisme calculateur, les oublis semicontrôlés du self-control, les escalades ou les raccourcis intuitifs des degrés de l'anticipation, les changements de règles du jeu en fonction du délai auquel un acteur envisage l'issue d'une interaction ${ }^{30}$, le désordonnement continuel des échelles de préférences au cours de la recherche d'un optimum, le recours à la vio-

Les figures subtiles de ce calcul qui rompt l'unité de l'acteur individuel font quelques uns des thèmes d'Ulysse et les sirènes (trad. Paris, Minuit, 1982) et du Laboureur et ses enfants: deux essais sur les limites de la rationalité (I. Elster trad. Paris, Minuit, 1986). 
lence ou à la menace, les renversements «du pour au contre» dont use «l'habile» pour donner raison au «peuple» contre la rationalité du «demi habile» («avec une idée de derrière la tête» comme disait Pascal ${ }^{31}$ ) ont toujours hanté les raisonnements des stratèges. De fait, on rencontre là des effets qui peuvent aussi bien être déduits du principe d'une rationalité stratégique que décrits à partir du principe de l'efficacité historique des conduites non-logiques. C'est pour avoir placé, au nom de la rationalité scientifique de l'explication, ce deuxième principe à la base de sa sociologie, que Pareto est paradoxalement passé, aux yeux de la plupart de ses commentateurs, pour un sociologue irrationaliste ou un laudateur de la violence.

\section{VI. ÉCONOMIE ET SOCIOLOGIE}

Pour les rapports entre calcul économique et analyse sociologique, tout commence là, Comme l'avait montré Pareto, on ne peut confondre l'utilité «pour un collectif » avec l'utilité «d'un collectif ${ }^{32}$.

\section{Utilité et désutilité des modèles utilitaristes}

Il est impossible dans un raisonnement utilitariste portant sur un collectif social, de passer, sans pétition de principe dans l'argumentaire ou erreur dans la démonstration, du calcul d'une «utilité pour» à l'affirmation d'une «utilité de». Cette impossibilité identifie une tâche explicative qui est précisément celle de la sociologie comme science historique. La sociologie vise en effet à comprendre et expliquer les actions sociales collectives telles qu'elles se sont manifestées historiquement dans la poursuite de certaines fins: pourquoi donc les fins de certaines fractions de la société ou de certains leaders ont-elles pesé plus lourd que d'autres? Le bilan des moyens qui ont assuré le succès d'une action ne s'épuise pas avec le bilan de l'inégalité des moyens économiques ou militaires dont dispose un acteur (individuel ou collectif), ni même avec le bilan des passions des uns et des autres. La ratiocination, c'est-à-dire l'usage biaisé du raisonnement la mauvaise foi intellectuelle, si l'on préfère - est une force sociale, chez le démagogue comme dans les raisonnements spontanées qu'une propagande ou une pastorale rencontrent dans un auditoire, surtout lorsque les deux ratiocinations coïncident, que ce soit par hasard ou par tactique, par méprise ou par communion: paniques ou croisades, révolutions ou exterminations ne puisent pas toute leur force mobilisatrice dans l'énergie aveugle des passions de masse, dans la seule affectivité irrationnelle des foules - dans les «psychoses » collectives, comme on dit aujourd'hui. Elles doivent l'essentiel de leur pouvoir de faire coaguler les convictions au fait d'en appeler au raisonnement. Comme le disait Pareto, « $\mathrm{Au}$ fond les hommes veulent raisonner: que ce soit bien ou mal peu importe $»^{33}$.

31 Pascal, Pensés, (1670), section V (édition Brunschwicg), Paris, 1976, pp. 135-145.

32 V. Pareto, Traité, op. cit., pour la comparaison entre le calcul de ces deux types d'ophélimité, qui se présente différemment en économie et en sociologie, pp. 1341-1344.

33 V. Pareto, Traité, op. cit., p. 527-528: «La grande importance de ce résidu engage à en faire un genre à part». Pareto en fait le «cinquième genre de la première classe» défini par «le besoin de logique (qui) est satisfait tant par une logique rigoureuse que par une pseudo-logique». 
La sociologie est indissociablement une science des rapports de force et une science de l'homo ratiocinans, puisque la justification pseudo-logique des rapports de force est une force. Ce n'est pas un calcul rationnel - que personne ne fait vraiment, chacun se contentant, comme le soulignait Pareto ${ }^{34}$, d'user de raisonnements pseudo-logiques pour emporter la décision ou l'accord collectif qui le favorise - qui permet de répondre à trois questions différentes trop souvent emmêlées : (a) une question politique, (b) une question historique, (c) une question sociologique.

(a) Pourquoi, dans un groupe ou une société, faudrait-il faire le choix d'une politique de guerre plutôt que de bien-être, de gloire nationale ou d'isolationnisme, de course à la puissance ou de gestion de la situation acquise ? Ou d'une politique de réduction des inégalités socio-économiques plutôt que d'une politique «libérale», qui produit le résultat inverse en soustrayant à toute régulation la concurrence inégale pour l'enrichissement? Une question de méthode se pose aussitôt: comment déterminer ce choix, si l'on veut le fonder sur un calcul de l'intérêt collectif? Peut-on faire reposer ce calcul sur le seul principe de rationalité ${ }^{35}$ ?

(b) Pourquoi un choix particulier, qu'on peut observer dans l'histoire, $a$ - $t$-il étéfait ou réitéré, en tel lieu et telle époque, sur tel de ces enjeux? Une autre question de méthode en découle: comment décrire et expliquer ce choix dans le déroulement des antécédents et conséquents par une méthode rationnelle d'imputation des effets à des causes?

(c) Pourquoi tels ou tels choix ont-ils réussi à rassembler les ressources psychologiques et économiques des membres ou des fractions d'un groupe ou d'une société? La question de méthode devient alors: comment décrire et interpréter la combinaison des motifs et des moyens qui ont été mis en œuvre dans un contexte particulier, pour comprendre rationnellement leur succès ou leur échec?

On n'utilise pas le principe de rationalité de la même manière selon qu'on essaie de répondre par un raisonnement rationnel à l'une ou l'autre de ces trois questions: la question (a) met en jeu la rationalité pratique d'une prise de décision; (b) et (c) sont des questions fort proches parce qu'elles appellent l'une et l'autre l'application d'une rationalité épistémique à l'explication comme à l'in-

34 Par exemple à propos des choix démographiques où «classes gouvernantes » et «classes gouvernées » se situent différemment par rapport à «l'utilité pour» et «l'utilité de»: «Seules les actions non-logiques sont capables de faire qu'en ces cas les classes gouvernées, oubliant le maximum d'utilité individuelle, se rapprochent du maximum d'utilité de la collectivité, ou bien seulement de celui de la classe gouvernante», TRAITE, pp. 1344-1345.

35 De Pareto à Arrow la réponse négative à cette question s'est précisée en même temps que la démonstration mathématique de l'impossibilité de déterminer par le seul calcul l'existence d'un optimum de l'utilité d'un collectif social.

36 Cf. pour cette distinction entre la «rationalité» d'un acte et la «rationalité» d'un raisonnement, G.G. Granger, «Les trois aspects de la rationalité économique», pp. 567-580 in Le modèle et l' enquête: les usages du principe de rationalité dans les sciences sociales (sous la dir. de L.-A. Gérard-Varet et J.-C. Passeron), Paris, Ed. EHESS, 1995. 
terprétation historiques; elles diffèrent cependant par la combinaison des raisons synchroniques ou diachroniques qui opèrent dans l'administration de leurs preuves: disons en gros comme sciences du récit et sciences de l'enquête.

La théorie économique peut se contenter d'enregistrer l'existence et la hiérarchie des préférences individuelles comme des utilités qu'elle constate après coup dans des transactions observables. À partir de là, elle a pu se construire en paradigme dans l'économie néo-classique et alimenter sur cette base axiomatique un main stream théorique qui fait aujourd'hui encore son prestige scientifique et son pouvoir de fascination sur les décideurs politiques. Pour sa part, la sociologie est engagée à faire comprendre la signification culturelle du choix des fins ou valeurs dans la société et contribuer ainsi à l'explication de leur émergence, de leur déclin ou de leur dominance historiques, tout en gardant une entière neutralité axiologique dans l'évaluation des diverses formes d'activité mentale qui les fondent. Il n'est pas étonnant que, même lorsqu'une science historique parvient à produire des connaissances sociologiques sur sa base scientifique propre, les intelligibilités disjointes selon lesquelles sont organisées ces connaissances puissent apparaître aux politiques ou à l'opinion publique comme autant de résultats contradictoires, démobilisateurs ou trop «relativistes ». Les sociologues qui ont aspiré au statut de conseillers du prince se sont vus plus souvent déboutés ou désavoués que les théoriciens de l'économie. Tant mieux, me semble-t-il, pour la sociologie, ainsi mieux immunisée contre la tentation axiologique; le prophétisme marxiste ne l'avait que trop habituée à céder à cette tentation.

\section{Les rapports entre la modélisation et la comparaison historique en sociologie}

Ce n'est pas dire que la méthodologie des sciences sociales puisse ou doive être étrangère aux méthodes des sciences expérimentales ou formelles. On y rencontre, assurément, des démarches hypothético-déductives (statistiques, mathématiques ou formalisées) qu'on peut même considérer comme les moments les plus sûrs du travail argumentatif dans nos disciplines. Mais les raisonnements appuyés sur ces méthodes n'introduisent dans les sciences historiques que des preuves conditionnelles, partielles et momentanées. L'apport démonstratif qu'elles autorisent reste tributaire d'un raisonnement interprétatif d'ensemble; il dépend d'une argumentation qui ne peut jamais être formalisée dans toute sa portée et tous les détails de sa morphologie probatoire. Pour répondre à une question de causalité ou pour interpréter, il faut toujours restituer les résultats d'un calcul aux concepts d'une intelligibilité sociologique, c'est-à-dire à un contexte historique $^{37}$. Et cela ne peut se faire que dans les mots d'une langue naturelle, puisque celle-ci peut seule véhiculer le contenu sémantique des interprétations historiques. Les modèles historiques les plus amples ou les plus mieux conceptualisés qui se construisent dans le domaine où la mesure est la plus facile, celui de l'histoire économique, restent des modèles à déïctiques.

37 Pour une analyse exemplifiée du sens formel et du sens historique des énoncés de la langue statistique dans un raisonnement sociologique, cf. J.-C. Passeron «Ce que dit un tableau et ce qu'on en dit», in : Le raisonnement sociologique, op. cit., p. 111-136. 
Lorsqu'une description oblige à passer d'une sémantique formelle à une sémantique empirique on cesse de raisonner en logicien pur. Un système formel ne peut qu'ignorer les significations théoriques et les protocoles descriptifs de la recherche empirique. La singularité d'un cas ou d'un contexte ne peut pas plus entrer dans un système formel que se retrouver dans l'universalité d'une déduction mathématique. Lorsqu'on entreprend l'analyse historique d'un processus, les informations exactes qui se composent dans l'explication ou l'interprétation deviennent nécessairement hétérogènes parle mode de «référer» qui commande leur signification. Le principe explicatif ne peut plus rester en ce cas celui d'une parfaite cohérence logique qui organiserait la structure interne de toutes les actions comme celles d'un acteur supposé rationnel. Le moteur explicatif des interactions devient celui d'une supputation instable, d'une inconstance stratégique des acteurs individuels ou collectifs. Pour le sociologue, les facteurs explicatifs d'une décision sont toujours susceptibles d'être pondérés différemment, en fonction du moment de l'interaction en cours et de son contexte, connu ou méconnu par les acteurs.

Il est, en somme, impossible au sociologue qui entend rester historien de construire les schémas conceptuels de ses explications comme des modèles de calcul. Au sens strict un modèle est l'algorithme d'un calcul qui a pour axiomes la commensurabilité et/ou la co-ordinabilité des éléments entrant dans le calcul. Il n'existe pas en sociologie de principes universels (pas de théorie pure) fondant la possibilité de composer ou de hiérarchiser éléments et mesures selon des règles qui resteraient constantes, d'une observation ou d'une enquête à l'autre. Pensons, pour seul exemple aux «coûts sociaux» (eux-mêmes peu commensurables entre eux, selon l'étalon auquel on les mesure) d'un système économique non-autorégulé comme l'est le marché capitaliste mondial. Comment faire entrer dans un calcul économique ou politique d'optimisation l'ensemble de toutes les «utilités » observables ou concevables, qui restent incommensurables par l'hétérogénéité des préférences qu'elles composent? Qu'on soit économiste, sociologue ou historien, on peut certes en raisonner de manière informée. On peut supputer, argumenter des balances raisonnables ou désirables entre les «coûts sociaux» et les «coûts économiques»d'une décision politique, mais on ne saurait calculer un optimum de cette balance et, encore moins, prévoir des valeurs à l'équilibre.

Ce qui interdit à l'acteur social de bâtir tous ses raisonnements sur la déduction ou l'expérimentation, ce qui interdit de même à la sociologie ou à toute science historique la prévision ou le chiffrage (a priori ou ex post) de la probabilité du succès d'une action, c'est, dans les deux cas, la structure même de la détermination des actions historiques. Ce n'est pas seulement que les probabilités ou les pondérations que le sociologue est condamné à introduire dans un raisonnement portant sur des faits datés et localisés soient approximatives - car il vaut toujours mieux calculer avec des valeurs approchées que sans ordres de grandeur. C'est plus fondamentalement que le sociologue ne dispose jamais, même quand il recourt aux méthodes quantitatives, d' «unités de mesure » et de règles de combinaison permettant de pondérer entre elles des données prélevées ou mesurées dans des systèmes partiels de préférences entre lesquels il ne peut généraliser les relations au-delà du contexte singulier où il les a observées. D'une aire culturelle ou d'une période de l'observation à une autre, les mesures ne sont ni substituables ni comparables sur la base d'une liste finie de traits désignés une fois pour toutes 
comme «pertinents» par une théorie qui encadrerait sans solution de continuité toutes les analyses comparatives.

Mais est-ce un mal méthodologique ou a-t-on affaire à une autre méthode? Les sciences historiques contrebalancent la discontinuité de leurs raisonnements quantitatifs et l'impureté logique de leurs preuves en multipliant les attendus comparatifs qui enrichissent la portée explicative de leur argumentation. Entendons par là que la valeur scientifique des assertions d'une enquête sera d'autant meilleure qu'elle aura réussi à formuler plus d'informations exactes dans un langage théorique mieux unifié par l'interprétation des actes: c'est la seule définition possible de l'argumentation scientifique dans une science de l'homme qui vise à la synthèse d'explications fondées sur des preuves de forme logique différente. Ce ne sont pas les méthodes qui différent entre des sciences sociales spécialisées comme l' économie, la démographie ou la linguistique, et des sciences sociales à visée synthétique comme l'histoire, l'anthropologie ou la sociologie: c'est leur argumentation d'ensemble qui articule différemment les mêmes méthodes de preuve pour construire une explication de forme historique comme un argumentaire composite: d'où la place différente que tiennent, dans leurs explications, la «théorie » pure, la formalisation, le calcul ou la modélisation, le terrain, le document, le vestige, le questionnement ou l'écoute des acteurs, bref le modèle et l'enquête. La discontinuité entre les assertions est le prix des savoirs de la complexité. L'appauvrissement des assertions historiques, le prix des savoirs formalisés.

\section{La grenouille et le scorpion : fable épistémologique}

Les sciences de l'enquête diffèrent fondamentalement des sciences du modèle par l'usage, exclusif ou non, qu'elles font du principe de rationalité pour expliquer les comportements sociaux. Le critère de la maximisation d'une «utilité individuelle», sur le quel reposent les modèles de l'économie mathématique ou de la théorie des jeux ne suffit jamais à définir la grammaire du langage théorique dont use un historien, un sociologue, un anthropologue. On peut ramasser cette différence dans l'historiette bien connue de la grenouille et du scorpion.

Un scorpion demande à une grenouille de le transporter sur son dos d'un bord à l'autre d'une rivière. La grenouille refuse au motif qu'elle risquerait d'être piquée à mort pendant la traversée. Argumentant et anticipant les effets avec l'habileté d'un philosophe «utilitariste» pour mieux atteindre son but, le scorpion lui répond qu'il n'y trouverait pour son compte aucun «intérêt» puisqu'il serait aussitôt noyé. Mais lorsque, au milieu de la rivière, la grenouille qui s'est laissé persuader par cette plaidoirie utilitariste s'étonne en mourant de la piqûre irrationnelle du scorpion, celui-ci peut répondre en soupirant, mais toujours aussi rationnellement par rapport à sa vérité vécue du moment: «Je n'y peux rien, c'est dans ma nature...»

Le scorpion suicidaire est le prototype du politique trompeur qui est trompé par sa propre habileté à improviser une plaidoirie à la fois logique et efficace. Il a réussi à persuader la grenouille et à la berner, mais il n'a pourtant pas eu une stratégie délibérée de mensonge, puisque le mensonge lui a été mortel. Dans l'histoire, il fait figure de virtuose du calcul utilitariste, floué par son propre raisonnement; et il est tout autant le dindon de cette farce logique que la grenouille, victime, elle, de sa croyance naïve en une rationalité trompeuse. La ratio- 
nalité de l'anticipation logique et le déterminisme biologique de l'instinct coexistent dans la fable, sans que le refus de la contradiction logique ait pu, à un moment ou à un autre de l'histoire, donner raison à l'un ou l'autre des deux acteurs. Tous deux ont fait assaut d' «anticipation rationnelle» comme disent les économistes, qui basent sur ce concept l'explication des décisions que des firmes prennent sur un «marché» en anticipant la stratégie prêtée à l'adversaire. Mais l'explication scientifique des actes du scorpion et de la grenouille doit se transformer à mesure que les causes de l'action de chacun changent en se succédant dans le temps. Dans un modèle, l'économiste fixe la logique du calcul en substituant le temps du modèle au temps historique; dans une enquête, le sociologue explore le devenir des interactions, en faisant varier ses méthodes par la mesure ou l'observation de terrain, par la comparaison historique ou statistique, afin de rendre probable une explication qui soit en même temps une interprétation plausible.

Le scorpion fait ici figure d'anti-Ulysse - si l'on pense à l'Ulysse d'Ulysse et les Sirènes ${ }^{38}$, capable d'anticiper, en son calcul rationnel, les faiblesses à venir d'un autre Ulysse, celui qui devra être lié au mât du navire et avoir les oreilles bouchées de cire pour ne pas entendre le chant des Sirènes et ne pas succomber à ses propres pulsions. A la différence de l'homo æeconomicus, l'homo sociologicus fait un usage de la rationalité presque toujours plus proche de celui du scorpion que de celui d'Ulysse. Là commence le partage méthodologique entre deux orientations les sciences sociales, celles du modèle et celles de l'enquête. On peut en effet interpréter de toutes sortes de manières la séquence d'interactions de l'historiette: on construit alors autant de théories explicatives de la catastrophe rationnelle survenue dans une interaction qui a pourtant échappé à des acteurs aspirant conjointement à un arbitrage rationnel de leurs décisions.

Demandons à la théorie d'un sociologue quel diable d'animal est ce scorpion. Dans sa typologie, Max Weber distinguait quatre «types purs de l'action sociale»: (I) «l'action rationnelle par rapport à des fins» qui en calcule les meilleurs moyens; (II) «l'action rationnelle par rapport à des valeurs » où le calcul se trouve limitée par un «commandement» inconditionnel; (III) «l'action traditionnelle» commandée sans calcul par l'autorité de qui s'est toujours fait ainsi; et (IV) «l'action affective » qui incline à l'obéissance par l'influence qu'exerce le «charisme » d'un chef, d'un prophète, d'une institution ou d'un livre sacré sur ceux qui reconnaissent sa légitimité. Le scorpion de la fable est wertrational (IIème type). C'est un calculateur rationnel, mais aveugle au coût, pourtant rationnellement prévisible dans le cours d'une psychanalyse, que lui fera consentir son désir de piquer un vivant trop proche, lorsque la pulsion instinctive interviendra en dépit de tout calcul comme une irrésistible «obligation interne». À la différence d'Ulysse il n'a pas su anticiper sa pulsion. L'inconditionnalité absolue des commandements instinctifs de l'espèce borne ici le calcul rationnel des moyens et des fins en empêchant toute supputation de leur valeur en fonction de leur coût $\mathrm{t}^{39}$.

Consultons maintenant un autre sociologue, Pareto par exemple: le scorpion se laisse alors comprendre comme un politique machiavélien. Il est prêt à utiliser

Cf. supra, I. Elster, Ulysse et les sirènes, op. cit.

39 M. Weber, Wirtschaft und Gesellschaft, Tübingen, JCB Mohr, 1922; trad. frcse, op cit. Cf. la définition de ces quatre types « purs » pp. 22-23. 
n'importe quel argument pour atteindre son objectif: persuader la grenouille de répondre à sa demande. Mais c'est un machiavélien imparfait, puisqu'il est incapable d'anticiper ou de contrôler ses propres réactions non-logiques comme il sait le faire pour manipuler les décisions d'une crédule grenouille. Reste un doute dans cette casuistique de la rationalité: si l'on se reporte aux classifications sociologiques de Pareto aussi précises que celles d'un entomologiste, le scorpion doitil figurer dans la première ou la deuxième espèce du «quatrième genre de la deuxième classe » des actions sociales ${ }^{40}$ ? Selon le critère parétien qui divise en deux le quatrième genre des actions non-logiques - celui de l'erreur de calcul ou de l'absence d'information - il faut trouver des indices pour trancher la question de savoir «s'il aurait accepté, ou non, le résultat objectif de sa stratégie au cas où il l'aurait connu».

En consultant sa bibliothèque des grandes œuvres théoriques, le lecteur imaginera sans peine d'autres lectures que wébériennes ou parétiennes de la stratégie du scorpion. Un scorpion pascalien, marxien, durkheimien, mertonien, darwinien, schumpétérien, aronien, statisticien, économètre, théoricien des jeux, simonien, braudellien, bourdieusien, elstérien, freudien, lacanien, herméneute, veynien, foucaldien, interactionniste ou ethnométhodologue laisserait tout aussi facilement interpréter sa rationalité catastrophique dans le cadre d'une théorie scientifique des actions ou interactions sociales. Il suffit, à chaque fois, de faire intervenir dans l'interprétation des actes successifs du scorpion les concepts théoriques qui redéfinissent, dès que le rationalisme utilitariste est à court de raisons, la place et la forme du principe de rationalité dans l'explication de ses actes.

On n'a que l'embarras du choix entre les explications: «chaînes d'imagination » aussi contraignantes que les «chaînes de nécessité » (chez Pascal) - «intérêt objectif de classe» et «idéologie» (chez Marx) - «contrainte sociale» et « anomie» (chez Durkheim) - «normativité » et «rôles sociaux» (chez Merton) «sélection naturelle» et «variation» (chez Darwin) - «périodicité de cycles nonisochrones » (chez Schumpeter) - «désillusions du progrès » ou «frustration relative» chez Aron - «degrés de signification statistique » d'une corrélation, mesurés par la distance entre «fréquence théorique » et «fréquence observée» (dans le calcul des probabilités) - «maximum», «optimum» ou équation mathématique (chez les économètres) - «équilibres de Nash», «coordination des joueurs » et «rationalité sous contraintes » (chez les théoriciens des jeux) - «rationalité procédurale» versus «rationalité substantive» chez Simon - «pesanteur du quotidien » versus «jeux de l'échange» chez Braudel - «système de domination » et «intériorisation de la nécessité » (chez Bourdieu) - «autonomie des normes » par rapport aux calculs d'utilité (chez Elster) - «ambivalence» des sentiments et « rationalisation» (chez Freud) - «leurre du désir» et «structure langagière de l'inconscient» (chez Lacan) - intrication de la «temporalité» et du «récit»chez Ricœur - «intrigue» historique versus poids de la «quotidianité» (chez Veyne) «diversité des scènes sociales» (chez Goffman) ou accoutability des comportements (chez Garfinkel).

40 Cf. la présentation par Pareto des classes, genres et espèces de l'action sociale dans le tableau synoptique de son Traité, op. cit., p. 67. 
Il y a toujours pour un acteur mille bonnes raisons, «compréhensibles » à un interprète rationnel, de n'être pas rationnel, au sens de la définition de la rationalité qui peut entrer dans un modèle de calcul. Il est clair, en tout cas, qu'un modèle formel ne fournit aucun moyen d'entrer dans les raisons des acteurs, et encore moins d'articuler les «causes» des actions sur les « raisons» des acteurs : il a fallu aux économistes - toute syntaxe exigeant une sémantique - doter à la va-vite leurs calculateurs fantômes d'une «psychologie de convention», qui n'explique que les décisions d'acteurs «purs» dans une «théorie pure». Dans les modèles de décision rationnelle, le décideur (individu ou firme) ne se contredit jamais, il cherche toujours à maximiser son intérêt; il recherche toujours le maximum d'information, il calcule au plus juste ce que coûte un moyen par rapport à un autre, il compare le coût de l'obtention d'une information ou de l'établissement d'une transaction en le rapportant aux conséquences qui découleraient du choix d'en faire l'économie: nous voilà bien loin de l'histoire des sociétés et des drames singuliers qui s'y nouent.

Mais attention! La pluralité de leurs théories ne condamne pas les sciences sociales au scepticisme; pas davantage à renoncer à la démarche scientifique. Si l'interprétation de l'historiette se prête indifféremment à toutes sortes d'herméneutiques, c'est précisément que l'interprète de l'action du scorpion ne dispose d'aucune autre données sur le contexte du «cas» que le récit d'une aventure unique. Aucune série d'indices ou de vestiges, pas de récits ou de sondages sur les rencontres entre scorpions et grenouilles: donc pas de possibilité de comparaison historique. Pas d'ethnographie ou d'écologie des fossés où cohabitent grenouilles et scorpions; aucune cartographie de leurs trajets et habitats. Pas de corpus: donc pas de recours possible aux méthodes statistiques; impossible de calculer la proportion des scorpions fossilisés au fonds des cours d'eau pour en comparer les variations à celles de la proportion des grenouilles qui y vivent; encore moins, dispose-t-on de protocoles d'observation ou de tests expérimentaux conduits sur des échantillons raisonnés ou représentatifs de scorpions ou de grenouilles. L'historien de cette rencontre sans lendemain entre une grenouille tombée du ciel et un scorpion miraculeux se trouve placé devant une histoire sans passé ni concomitants.

Dans une tâche analogue - tenir et améliorer des raisonnements explicatifs les sciences historiques ne sont pas aussi démunies. Elles disposent d'une panoplie de méthodes et de schèmes de raisonnement permettant de composer ces méthodes pour traiter leurs données d'observation et les interpréter dans une argumentation explicative: «variations concomitantes », «simulation » des effets dans un modèle, «tests de signification» pour chiffrer la probabilité d'une interaction entre variables, algorithmes de calcul fournis par les statistiques «descriptive» ou «analytique», échantillonnage et enquête de terrain. Mais aussi comparaison historique de faits «analogues » ou «contrastés » prélevés dans des contextes proches ou éloignés, critique interne et externe de documents écrits ou oraux, «analyse structurale» des textes ou analyse «pragmatique» des situations de parole, mise en série de vestiges, essais de langages conceptuels différents pour comparer leur force probatoire dans l'explication et l'interprétation etc. Toutes ces méthodes organisent des «styles d'argumentation» fort différents, qui fondent à leur tour des styles d'interprétation, jamais complètement traduisibles l'un dans l'autre, par lesquels des théories différentes construisent différemment leurs faits. Une expli- 
cation économique ne réfute pas une explication sociologique, et vice-versa; ni une interprétation psychanalytique une interprétation anthropologique. Ce qui caractérise le statut épistémologique des sciences sociales c'est que leurs raisonnements ne peuvent transmettre la vérité d'une proposition à la suivante comme dans une chaîne déductive. Mais ces sciences ont en commun une autre manière de prouver: faire converger des preuves de forme logique différente dans un argumentaire d'ensemble, leurs arguments dans un langage de l'interprétation, leurs interprétations dans une théorie plausible.

Tout comme les interprétations multiples de la stratégie d'un scorpion qui, pour traverser une rivière, a trouvé malgré lui le moyen de se suicider en toute rationalité avec la complicité d'une grenouille qui s'est laissé assassiner pour avoir trop fait confiance à la validité universelle des calculs d'utilité, les théories interprétatives des sciences sociales restent inévitablement multiples et concurrentes. Leurs données d'observation ne permettent jamais, en effet, de les départager ni par une démonstration logico-mathématique qui opérerait comme dans un pur système formel, ni par un raisonnement expérimental qui pourrait être mené de bout en bout «toutes choses égales par ailleurs». Pourtant, selon les «cas» considérés, leurs mesures ou leurs estimations quantitatives, leurs descriptions ou leurs argumentations, leurs généralisations théoriques ou leurs présomptions explicatives ne sont pas équivalentes. Les théories fondées sur l'enquête historique sont inégalement probantes, leurs intelligibilités sont inégalement robustes selon la pertinence des matériaux empiriques qu'elles ont rassemblés et selon le style de preuve qui soude dans un argumentaire leurs méthodes de traitement des données.

Les sciences historiques sont des sciences où faire preuve n'est jamais une simple question de tout ou rien, comme dans un jeu à somme nulle où une proposition démontrée ne peut gagner sa vérité que pour autant que la proposition contradictoire y perd toute la sienne par l'efficace de cette même démonstration. C'est une question de plus ou de moins dans «l'allongement du questionnaire». Les réponses au questionnaire que le chercheur administre à un objet historique sont d'autant mieux descriptives qu'elles sont plus détaillées. Mais elles ne deviennent plus véridiques que si les questions auxquelles elles répondent sont devenues en même temps plus pertinentes pour le sens du questionnement. L'amélioration des explications ne peut, ni théoriquement ni empiriquement, être ici séparée de la valeur des interprétations qu'elles apportent. La «densification de la description», son caractère plus ou moins «fouillé », est condition nécessaire mais non suffisante de l'amélioration de la preuve, puisque la force de celle-ci ne peut elle-même être évaluée qu'en fonction de l'adéquation de l'argumentaire au cas singulier. Dans le langage épistémologique qu'employait Max Weber, l' «adéquation causale» d'une assertion historique n'est pas séparable de son «adéquation quant au sens »: cette caractéristique, valable dans toutes les sciences historiques, qui sont à la fois des sciences de l'enquête et des sciences de l'interprétation, explique les possibilités de renouvellement indéfini de la recherche historique. Que l'analyse historique soit interminable ne prouve rien contre sa scientificité, pas plus que le caractère «interminable» d'une analyse de l'inconscient ne fait de la psychanalyse une science de chimère:

«Il y a des sciences auxquelles il a été donné de rester éternellement jeunes. C'est le cas de toutes les disciplines historiques, de toutes celles que le flux éter- 
nellement mouvant de la culture alimente sans cesse en nouvelles problématiques. $A u$ cour de leur tâche sont inscrits en même temps le caractère provisoire de toutes les constructions idéal-typiques et la nécessité inéluctable d'en construire de toujours nouvelles.» ${ }^{41}$

Fort heureusement pour «l'intérêt» scientifique des recherches menées dans les sciences sociales, l'histoire des sociétés humaines n'offre pas aux chercheurs des données aussi raréfiées que l'historiette romanesque du scorpion, diplômé en économie à Princeton, brillant avocat de l'anticipation rationnelle, mais incapable de l'intuition non-logique - ou d'un rien de psychanalyse - qui lui aurait permis de suspecter la duplicité de ses règles de décision pour anticiper un peu plus «raisonnablement» les risques de son talent de calculateur.

Ecole des Hautes Etudes en Sciences sociales

Paris-Marseille

41 M. Weber, Gesammelte Aufsätze zur Wissenschaftslehre, Tübingen, JCB Mohr, 1922, trad. mod.; trad. fr. J. Freund, Paris, Plon, 1965, p. 202. 